\title{
Studying the kinematic asymmetries of disks and post-coalescence mergers using a new "kinemetry" criterion
}

\author{
E. Bellocchi, S. Arribas, and L. Colina
}

Centro de Astrobiología, Departamento de Astrofísica, CSIC-INTA, Cra. de Ajalvir Km. 4, 28850 - Torrejón de Ardoz, Madrid, Spain e-mail: bellochie@cab.inta-csic.es

Received 16 August 2011/ Accepted 6 March 2012

\begin{abstract}
Context. Ultra luminous and luminous infrared galaxies [(U)LIRGs] are important galaxy populations for studying galaxy evolution, and are likely to have been responsible for a significant fraction of the star formation that occurred prior to $z \sim 1$. Local (U)LIRGs can be used to study criteria that are suitable for characterizing similar high redshift populations. We are particularly interested in identifying reliable kinematic-based methods capable of distinguishing disks and mergers, as their relative fraction is a key observational input to constrain different evolutionary scenarios.

Aims. Our goal is to analyze in detail the kinematics of the ionized gas of a small sample of LIRGs and study criteria that permit us to characterize the evolutionary status of these systems.

Methods. We obtained Very Large Telescope VIMOS optical integral field spectroscopy (IFS) data of four LIRGs selected at similar distances $(\sim 70 \mathrm{Mpc})$ to avoid relative resolution effects. Two of these systems had been previously classified as regular isolated disks galaxies and the other two as post-coalescence mergers based on their morphology. The kinemetry method (developed by Krajnović and coworkers) is used to characterize the kinematic properties of these galaxies and discuss new criteria for distinguishing their status.

Results. We present and discuss new kinematic maps (i.e., velocity field and velocity dispersion) for these four galaxies. These kinematic data suggest that nuclear outflows exist in all these galaxies, and are particularly intense for the post-coalescence merger systems. The $v_{\mathrm{c}} / \sigma_{\mathrm{c}}$ parameter has values between those that are typical of local spiral galaxies (i.e., $\left.v_{\mathrm{c}} / \sigma_{\mathrm{c}}=5-15\right)$ and those obtained for Lyman break analogs at $z \sim 0.2$ (i.e., $v_{\mathrm{c}} / \sigma_{\mathrm{c}}=0.4-1.8$ ). Our use of one-dimensional parameters, such as $v_{\mathrm{c}} / \sigma_{\mathrm{c}}$ or $v_{\text {shear }} / \Sigma$, does not allow us to distinguish between the two groups (i.e., disks, post-coalescence systems). However, when the full two-dimensional kinematic information of the IFS data is analyzed by means of kinemetry, their morphological and kinematic classifications are consistent, with disks having lower kinematic asymmetries than post-coalescence mergers. We propose and discuss a new kinematic criterion to differentiate between these two groups. In particular, we introduce a weighting that favors the outer parts of the kinematic maps when computing the total asymmetries. This step is taken because post-coalescence mergers display relatively small kinematic asymmetries in their inner parts as a consequence of the rapid relaxation of gas into a rotating disk, whereas the outer parts continue to be out of equilibrium (i.e., to have larger asymmetries). We find that, in addition to distinguishing post-coalescence mergers from rotating disks, this new criterion has the advantage of being less sensitive to angular resolution effects. According to previous kinemetry-based analyses designed to distinguish disks and mergers at high- $z$, the present post-coalescence systems would have been classified as disks. This indicates that the separation of disks from mergers depends on the definition of a merger. It also suggests that previous estimates of the merger/disk ratio might have been underestimated, but larger samples are necessary to establish a firmer conclusion.
\end{abstract}

Key words. galaxies: kinematics and dynamics - instrumentation: spectrographs - galaxies: structure - galaxies: interactions

\section{Introduction}

The kinematic characterization of high redshift (high-z) galaxy populations is a key observational stage in attempting to distinguish between different galaxy evolutionary scenarios, since it helps us to determine the number ratio of rotating disks to mergers at different cosmic epochs. It also provides a way of constraining the relative roles of major mergers and steady cool gas accretion in shaping galaxies, which remains a topic of discussion (e.g. Genzel et al. 2001; Tacconi et al. 2008; Dekel et al. 2009; Förster Schreiber et al. 2009; Lemoine-Busserolle \& Lamareille 2010; Lemoine-Busserolle et al. 2010; Bournaud et al. 2011; Epinat 2011).

With the aid of integral field spectroscopy (IFS), we can resolve the kinematical status and internal processes at work within galaxies and more clearly understand the role of the dominant mechanisms involved at early epochs of galaxy formation. For instance, Förster Schreiber et al. (2009) classified the SINS (Spectroscopic Imaging survey in the Near-infrared with SINFONI) sample (i.e., 63 galaxies with $1.3<z<$ 2.6) concluding that these objects can be classified as either "rotation-dominated", interacting/merging or "dispersiondominated" (i.e., dominated by large amounts of random motions) systems. They found that galaxies in the SINS sample can be evenly divided between each group (i.e., one-third each). They found that a small fraction of galaxies display signs of major merger events (i.e., Förster Schreiber et al. 2006; Law et al. 2009; Genzel et al. 2006, 2008), which thus implied that the majority of star-forming galaxies at these redshifts are fed by gas via continuous cold flows along streams (including minor mergers).

At intermediate redshift $(z \sim 0.6)$, spatially resolved kinematics has revealed that a large fraction of galaxies have chaotic velocity fields, which indicate that most local spirals have been rebuilt after a major merger since $z=1$ (Hammer et al. 2005; Puech 2010). At similar redshifts, Flores et al. (2006) 
and Yang et al. (2008) investigated the velocity fields of galaxies with IFS, finding that only $35 \%$ of their sources can be classified as rotating disks while the reminder show perturbed or complex kinematics.

Some local galaxy populations are particularly relevant to the study of galaxy evolution, such as nearby luminous and ultraluminous infrared galaxies (LIRGs, $L_{\mathrm{IR}}=[8-1000 \mu \mathrm{m}]=$ $10^{11}-10^{12} L_{\odot}$, and ULIRGs, $L_{\mathrm{IR}}>10^{12} L_{\odot}$, respectively). Despite these objects being relatively rare in the local universe, they are far more numerous at high- $z$ and responsible for a significant fraction of previous star formation prior to redshift $z \sim 1$ (e.g., Le Floc'h et al. 2005; Pérez-González et al. 2005, 2008). These objects exhibit a wide range of interacting/merging properties, ranging from mostly isolated disks for low luminosity LIRGs (e.g. $\log L_{\mathrm{IR}} \leq 11.3$, Arribas et al. 2004) to mainly merger remnants for ULIRGs (e.g. $\log L_{\mathrm{IR}} \geq 12$, Veilleux et al. 2002). Local (U)LIRGs were initially assumed to be the local counterpart of high-z (U)LIRGs similar to those discovered by Spitzer and the more luminous submillimeter galaxies (SMGs; e.g. Blain et al. 2002; Tacconi et al. 2006). The study of local (U)LIRGs is also important to improve our understanding of star formation history in the universe and permits us to correlate the properties of local and high- $z$ populations. Moreover, large-scale turbulences, tidal tails, and outflows can be studied in detail allowing a good characterization of these objects. However, there has been some discussion about the similarities between local and distant (U)LIRGs (e.g., Rujopakarn et al. 2011, and references therein).

Several authors have analyzed the velocity fields and velocity dispersion maps of different galaxy samples with the aim of discerning merging and non-merging systems based on their kinematic properties using the kinemetry methodology (Krajnović et al. 2006). Jesseit et al. (2007) and Kronberger et al. (2007) investigated the distortions in the velocity fields of simulated interacting disk/merger galaxies at different redshifts in the range $z=0-1$, while Shapiro et al. (2008, hereafter, S08), developed a straightforward means of classifying $z \sim 2$ galaxies, observed with SINFONI/VLT, as disks or mergers based on the asymmetries of their velocity field and velocity dispersion maps. They compared and distinguished two kinematic classes characterized by recent major merger events (i.e., mergers) and the absence of signs of interacting or recent merger activity (i.e., disks).

Similar works based on IFS data (i.e., OSIRIS/Keck, Basu-Zych et al. 2009; Gonçalves et al. 2010) have focused on nearby objects (i.e., supercompact UV-luminous galaxies (ScUVLGs) and Lyman break analogs (LBAs)). The morphology and kinematics of LBAs projected at high redshift are similar to those of compact and dispersion-dominated $z \sim 2$ galaxies (Basu-Zych et al. 2009). This suggests that galaxy interactions and/or mergers might also drive the dynamics of dispersiondominated $z \sim 2$ galaxies.

In this paper, we present new spatially resolved kinematics of four local LIRGs obtained with the VLT/VIMOS IFU as part of a larger project to characterize the properties of (U)LIRGs based on optical and infrared IFS data (Colina et al. 2005; Arribas et al. 2008; Monreal-Ibero et al. 2010; Rodríguez-Zaurín et al. 2011, and references therein). Two of our post-coalescence mergers will offer the opportunity to explore the potential of the kinemetry method when analyzing the velocity fields and velocity dispersion maps in moderately disturbed and partially relaxed systems. The paper is structured as follows. In Sect. 2, we present the sample selection as well as some details about the observations, data reductions, line fitting, and velocity map construction. Section 3 is devoted to the description of the main kinematic properties of the individual objects. In Sect. 4, the kinemetry analysis and its potential to distinguish disks and mergers are presented and discussed with the aid of different asymmetry planes. Finally, the main results and conclusions are summarized in Sect. 5.

Throughout the paper, we assume that $H_{0}=$ $70 \mathrm{~km} \mathrm{~s}^{-1} \mathrm{Mpc}^{-1}, \Omega_{\mathrm{M}}=0.3$, and $\Omega_{\Lambda}=0.7$.

\section{The VIMOS sample, observations, data reduction, line fitting, and map construction}

\subsection{The sample and morphological classification}

For the present analysis, we selected four LIRG galaxies at similar distances ( 70 Mpc): two of them (i.e., IRAS F11255-4120, IRAS F10567-4310) had been morphologically classified as class 0 objects and two (i.e., IRAS F04315-0840, IRAS F214533511) as class 2 objects according to Arribas et al. (2008) and Rodríguez Zaurín et al. (2010, hereafter, Paper I and Paper III respectively). In this classification scheme, class 0 is defined as an object with a relatively symmetric morphology which appears to be isolated with no evidence of either strong past or ongoing interaction (hereafter, disks), while class 2 is an object with a morphology that is indicative of a post-coalescence merging phase, with a single nucleus or two distinct nuclei at a projected distance $D<1.5 \mathrm{kpc}$ (hereafter, post-coalescence mergers). We chose these four systems to be at the same distance as they allow us to discuss their relative kinematic properties avoiding any problems caused by variations in linear resolution. In Table 1, we present the main properties of the sample, noting that the systems classified as post-coalescence mergers have higher $L_{\mathrm{IR}}$ than the disks.

\subsection{Observations}

The observations were carried out in service mode using the Integral Field Unit of VIMOS (Le Fèvre et al. 2003), at the Very Large Telescope (VLT), covering the spectral range (5250-7400) $\AA$ with the high resolution mode "HR-orange" (grating GG435) and a mean spectral resolution of 3470 . The field of view (FoV) in this configuration is $27^{\prime \prime} \times 27^{\prime \prime}$, with a spaxel scale of $0.67^{\prime \prime}$ per fiber (i.e., 1600 spectra are obtained simultaneously from $40 \times 40$ fibers array). A square four pointing dithering pattern is used, with a relative offset of $2.7^{\prime \prime}$ (i.e., four spaxels). The exposure time per pointing ranges from 720 to $850 \mathrm{~s}$, such that, the total integration time per galaxy is between 2880 and 3400 s. For further details about the observations, we refer to Paper I.

\subsection{Data reduction}

The VIMOS data are reduced with a combination of the pipeline Esorex (version 3.5.1 and 3.6.5) included in the pipeline provided by ESO, and different customized IDL and IRAF scripts. The basic data reduction is performed using the Esorex pipeline (bias subtraction, flat-field correction, spectral tracing and extraction, correction of fiber and pixel transmission, and relative flux calibration). Four quadrants per pointing are then reduced individually and combined to produce a single data cube associated each pointing. After that, the four independent dithered pointings are combined together to produce the final "super-cube", containing $44 \times 44$ spaxels for each object (i.e., 
Table 1. General properties of the LIRG sub-sample.

\begin{tabular}{lccccccccc}
\hline \hline $\begin{array}{l}\text { ID1 } \\
\text { IRAS }\end{array}$ & $\begin{array}{c}\text { ID2 } \\
\text { Other }\end{array}$ & $\begin{array}{c}\alpha \\
(\mathrm{J} 2000)\end{array}$ & $\begin{array}{c}\delta \\
(\mathrm{J} 2000)\end{array}$ & $z$ & $\begin{array}{c}D \\
(\mathrm{Mpc})\end{array}$ & $\begin{array}{c}\text { Scale } \\
(\mathrm{pc} / \mathrm{arcsec}) \\
(7)\end{array}$ & $\begin{array}{c}\log L_{\text {IR }} \\
\left(L_{\odot}\right)\end{array}$ & $\begin{array}{c}\text { Class } \\
(8)\end{array}$ & $\begin{array}{c}\text { References } \\
(9)\end{array}$ \\
\hline F11255-4120 & ESO 319-G022 & $11: 27: 54.1$ & $-41: 36: 52$ & 0.016351 & 70.9 & 333 & 11.04 & 0 & 1 \\
F10567-4310 & ESO 264-G057 & $10: 59: 01.8$ & $-43: 26: 26$ & 0.017199 & 74.6 & 350 & 11.07 & 0 & 1 \\
F04315-0840 & NGC 1614 & $04: 33: 59.8$ & $-08: 34: 44$ & 0.015983 & 69.1 & 325 & 11.69 & 2 & 1,2 \\
F21453-3511 & NGC 7130 & $21: 48: 19.5$ & $-34: 57: 05$ & 0.016151 & 70.0 & 329 & 11.41 & 2 & 1,2 \\
\hline
\end{tabular}

Notes. Column (1): object designation in the IRAS faint source catalogue (FSC). Column (2): other name. Columns (3) and (4): right ascension (hours, minutes, and seconds) and declination (degrees, arcminutes, and arcseconds) from the IRAS FSC. Column (5): redshift of the IRAS sources from the NASA Extragalactic Database (NED). Column (6): luminosity distances assuming a $\Lambda$ DCM cosmology with $H_{0}=70 \mathrm{~km} \mathrm{~s}^{-1} \mathrm{Mpc}^{-1}, \Omega_{\mathrm{M}}=0.3$ and $\Omega_{\Lambda}=0.7$, using the Edward L. Wright Cosmology calculator. Column (7): scale. Column (8): infrared luminosity $\left(L_{\mathrm{IR}}=L(8-1000) \mu \mathrm{m}\right)$, in units of solar bolometric luminosity, calculated using the fluxes in the four IRAS bands as given in Sanders et al. (2003) when available. Otherwise we use the standard prescription in Sanders \& Mirabel (1996) with the values in the IRAS point and faint source catalogues. Column (9): morphology class. For further information, see Table 1 in Rodríguez Zaurín et al. (2010).

References. (1) Veilleux et al. (1995); (2) Corbett et al. (2003).

Table 2. Results of the calibration check using the [OI] $\lambda 6300.3 \AA$ sky line.

\begin{tabular}{ccc}
\hline \hline IRAS Galaxy & $\left\langle\lambda_{\mathrm{c}}\right\rangle \pm \Delta \lambda_{\mathrm{c}}[\AA]$ & $\left\langle\sigma_{\text {INS }}\right\rangle \pm$ std $[\AA]$ \\
\hline F11255-4120 & $6300.42 \pm 0.15$ & $0.78 \pm 0.09$ \\
F10567-4310 & $6300.17 \pm 0.13$ & $0.77 \pm 0.09$ \\
F04315-0840 & $6300.24 \pm 0.10$ & $0.78 \pm 0.08$ \\
F21453-3511 & $6300.27 \pm 0.11$ & $0.77 \pm 0.06$ \\
\hline
\end{tabular}

Notes. Typical values of the central wavelength and width distribution of the [OI] $\lambda 6300.3 \AA$ sky line registered in the data cubes along with their standard deviations (errors).

1936 spectra). Further details about the data reduction for the whole sample can be found in Paper I and Paper III.

The wavelength calibration, the instrumental profile, and fiber-to-fiber transmission correction are checked for the four galaxies using the $[\mathrm{OI}] \lambda 6300.3 \AA$ sky line as in Paper I. Our interest is focused on the $\mathrm{H} \alpha$ and [NII] $\lambda \lambda 6548.1,6583.4 \AA$ emission lines, hence the sky line here considered is suitable because of its proximity to these lines. In Table 2 , the average values for the central wavelength $\left\langle\lambda_{\mathrm{c}}\right\rangle$ and (instrumental) width $\left\langle\sigma_{\text {INS }}\right\rangle$, with their standard deviations, are shown for the four galaxies.

\subsection{Line fitting and map construction}

As mentioned in the previous paragraph, we are interested in the $\mathrm{H} \alpha-[\mathrm{NII}]$ emission-line complex analysis. The observed emission profiles of the individual spectra are fitted with a Gaussian function using an IDL routine (i.e., MPFITEXPR, implemented by Markwardt). This algorithm finds the optimal set of model parameters, which matches the data and is able to fix the line intensity ratios and the wavelength differences according to the atomic parameters when adjusting multiple emission lines (i.e., the $\mathrm{H} \alpha-[\mathrm{NII}]$ complex). The MPFIT routine evaluates the errors in the output parameters and gives an estimate of the goodness of the fit. For each emission line, we end up with the following information: central wavelength $\left(\lambda_{\mathrm{c}}\right)$, intrinsic width $\left(\sigma_{\text {line }}, \sigma_{\text {line }}=\sqrt{\sigma_{\text {obs }}^{2}-\sigma_{\text {INS }}^{2}}\right)$, and flux intensity along with their respective errors. To obtain the radial-velocity uncertainty estimates, wavelength calibration and fitting errors were combined in quadrature, giving a global wavelength error of $\Delta \lambda_{\mathrm{tot}}=\sqrt{\Delta \lambda_{\mathrm{MPFIT}}^{2}+\Delta \lambda_{\mathrm{c}}^{2}}$. For high signal-to-noise ratio $(\mathrm{S} / \mathrm{N})$ spectra, the wavelength calibration error can be considered the main source of uncertainty because the fitting errors are typically substantially smaller (and vice versa for low $\mathrm{S} / \mathrm{N}$ spectra). For the fitting errors, we derive values that are generally smaller than $0.2 \AA$, while the wavelength calibration errors are on the order of $\sim 0.12 \AA$ as shown in Table 2 . An average value of the $\sigma_{\text {INS }}$ of $(37 \pm 5) \mathrm{km} \mathrm{s}^{-1}$ is derived at the corresponding wavelength, along with a mean FWHM of $(1.82 \pm 0.19) \AA$.

We start fitting all the lines to single Gaussian profiles because the main component usually extends over the entire galaxy. Two-Gaussian profiles (i.e., main and broad components) are needed to describe the inner regions of the four objects. Using a collection of procedures written in IDL code (i.e., jmaplot, Maíz-Apellániz 2004), we generate flux intensity, velocity field, and velocity dispersion maps, respectively, for the main and broad components (Figs. 1-4) along with a VIMOS continuum image. When HST images are available (i.e., acquired by NICMOS F160W ( $H$-band) and ACS $F 814 W$ $(I$ band $))$, they are also shown in the panels.

\section{Kinematic properties of individual objects}

We describe the global kinematic behavior inferred from our IFS maps (i.e., flux intensity, velocity field, and velocity dispersion). We also determined some kinematic parameters (e.g., $\sigma_{\mathrm{c}}, v_{\mathrm{c}} / \sigma_{\mathrm{c}}$, $v_{\text {shear }} / \Sigma$, and dynamical mass $M_{\text {dyn }}$ ) that help us to characterize these systems.

The ratio of the maximum circular rotation velocity $v_{\mathrm{c}}$ (i.e., the half of the observed peak-to-peak velocity) to the central velocity dispersion $\sigma_{\mathrm{c}}$ measures the amount of gravitational support of a system in equilibrium. A value of $v_{\mathrm{c}} / \sigma_{\mathrm{c}} \geq 1$ is the signature of a rotation-dominated system, while a lower value (i.e., $v_{\mathrm{c}} / \sigma_{\mathrm{c}}<1$ ) means that the object is dispersion-dominated, as in the case of elliptical galaxies, where the galaxy resist to gravitational collapse because of the pressure produced by the random motions of the stars. When analyzing the $v_{\mathrm{c}} / \sigma_{\mathrm{c}}$ parameter, it is important to realize that the presence of flows, superwinds, or other merger-induced processes are factors that may lead to large $v_{\mathrm{c}}$ or $\sigma_{\mathrm{c}}$ values. We therefore compute the circular velocity $v_{\mathrm{c}}$ and the central velocity dispersion $\sigma_{\mathrm{c}}$ using the component of one-Gaussian model fit (i.e., 1c) and the main (i.e., the one defining the systemic behavior) component of the twoGaussian model fit (i.e., 2c). 
Source F11255-4120
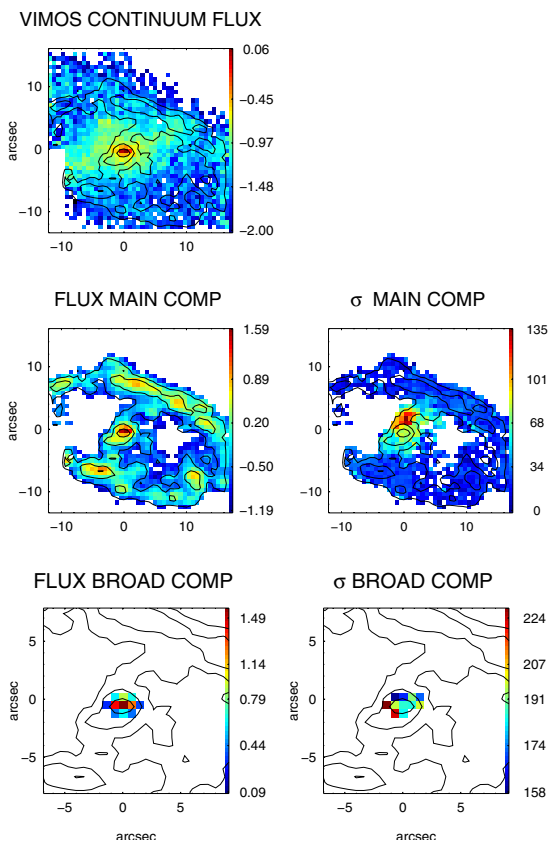

$\left(0.67^{\prime \prime}, 0^{\prime \prime}\right)$
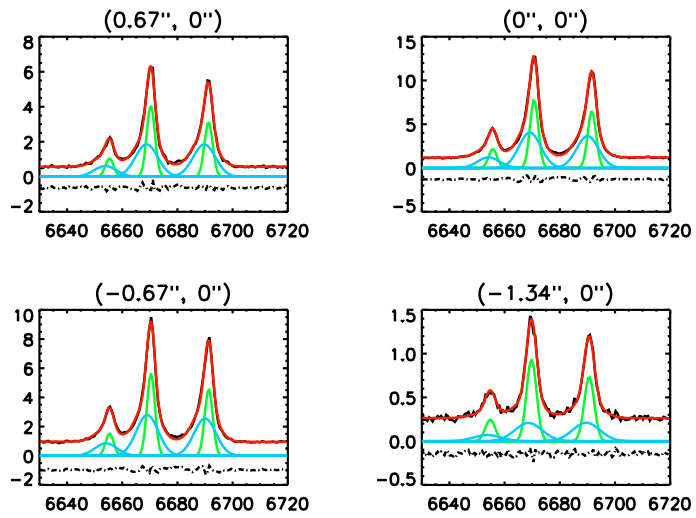

Fig. 1. Top panel: upper row: $\mathrm{H} \alpha$ maps for the class 0 object IRAS F11255-4120. VIMOS continuum image; HST continuum images are unavailable for this galaxy. Middle row: the flux intensity, velocity dispersion $\sigma\left(\mathrm{km} \mathrm{s}^{-1}\right)$, and velocity field $v$ maps $\left(\mathrm{km} \mathrm{s}^{-1}\right)$ for the main component. Lower row: respective maps for the broad component. Note the different FoV between the main and the broad components. The latter has been zoomed because it covers a small area. The flux intensity maps are represented on a logarithmic scale and in arbitrary flux units. All the images are centered using the $\mathrm{H} \alpha$ flux intensity peak and the iso-contours of the $\mathrm{H} \alpha$ flux are overplotted. Bottom panel: $\mathrm{H} \alpha$ [NII] observed spectra of IRAS F11255-4120 for selected inner regions (indicated by the coordinates in the top label using the same reference system as in the top panel) where the main and broad components coexist. The red curve shows the total $\mathrm{H} \alpha$-[NII] components obtained from multi-component Gaussian fits. The green and blue curves represent, respectively, the main and broad components.

Since class 0 objects have the characteristics of an ideal rotating disk ${ }^{1}$ we correct their kinematic values for their respective inclinations; class 2 objects have velocity fields that are distorted such that we derive their kinematic values with and without correcting for the inclination.

\footnotetext{
1 We refer to an ideal rotating disk as a thin disk with gas clouds kinematically characterized by a velocity field that peaks at the galaxy major axis and goes to zero along the minor axis of each orbit, while the velocity dispersion remains constant along each orbit and decreases between orbits of increasing major axes (see description in Sect. 4.1).
}

Source F10567-4310
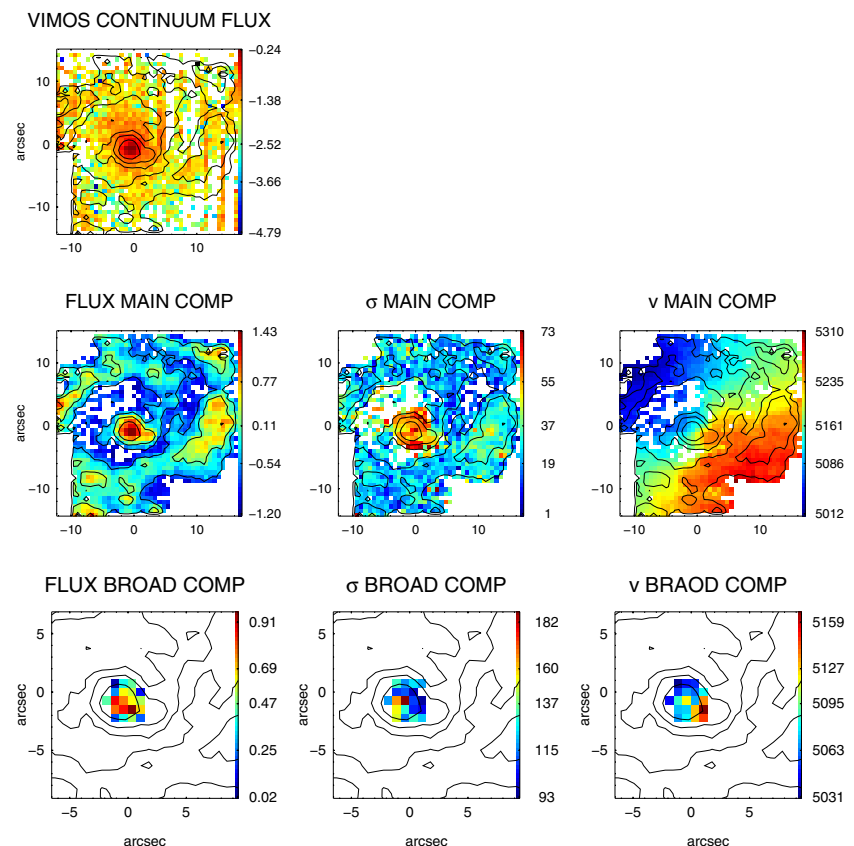

$\sigma$ BROAD COMP

v BRAOD COMP
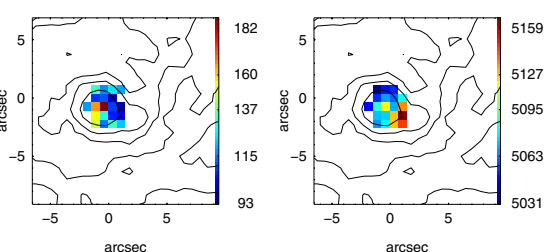

$\left(1.34^{\prime \prime}, 0^{\prime \prime}\right)$

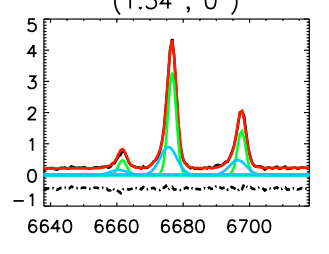

$\left(0.67^{\prime \prime}, 0^{\prime \prime}\right)$
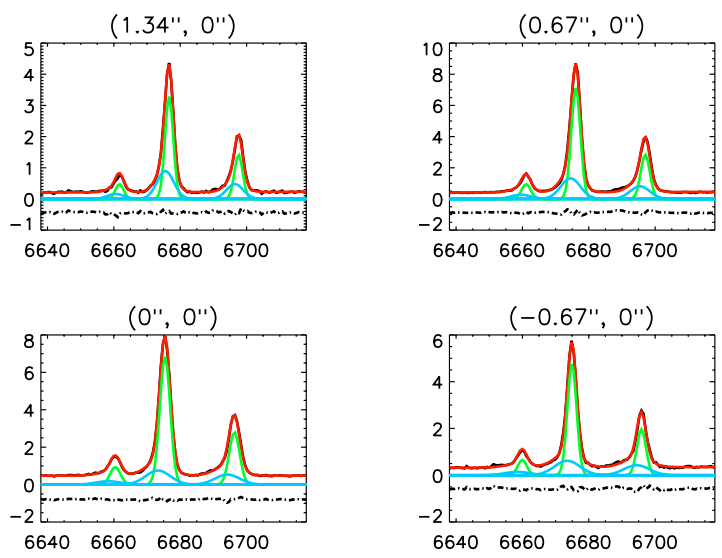

Fig. 2. Top panel: upper row: $\mathrm{H} \alpha$ maps for the class 0 object IRAS F10567-4310. VIMOS continuum image; HST continuum images are unavailable for this galaxy. Middle row: the flux intensity, velocity dispersion $\sigma\left(\mathrm{km} \mathrm{s}^{-1}\right)$, and velocity field $v$ maps $\left(\mathrm{km} \mathrm{s}^{-1}\right)$ for the main component. Lower row: respective maps for the broad component. Note the different FoV between the main and the broad components. The latter has been zoomed because it covers a small area. The flux intensity maps are represented on a logarithmic scale and in arbitrary flux units. All the images are centered using the $\mathrm{H}_{\alpha}$ flux intensity peak and the iso-contours of the $\mathrm{H}_{\alpha}$ flux are overplotted. Bottom panel: $\mathrm{H}_{\alpha}$-[NII] observed spectra of IRAS F10567-4310 for selected inner regions (indicated by the coordinates in the top label using the same reference system as in the top panel) where the main and broad components coexist. The red curve shows the total $\mathrm{H} \alpha$-[NII] components obtained from multi-component Gaussian fits. The green and blue curves represent, respectively, the main and broad components.

We derived the ratio of the velocity shear $v_{\text {shear }}$ to the global velocity dispersion in the whole galaxy $\Sigma^{2}$, as done in Gonçalves et al. (2010). The $v_{\text {shear }}$ (uncorrected for the inclination of the object) was computed as the median of the 5-percentile at each end of the velocity distribution, for the values of $v_{\max }$ and $v_{\min }$ and then defined as $v_{\text {shear }}=\frac{1}{2}\left(v_{\max }-v_{\min }\right)$.

\footnotetext{
${ }^{2}$ The global velocity dispersion $\Sigma$ of each object is measured as the mean value over all the spaxels.
} 
Source F04315-0840
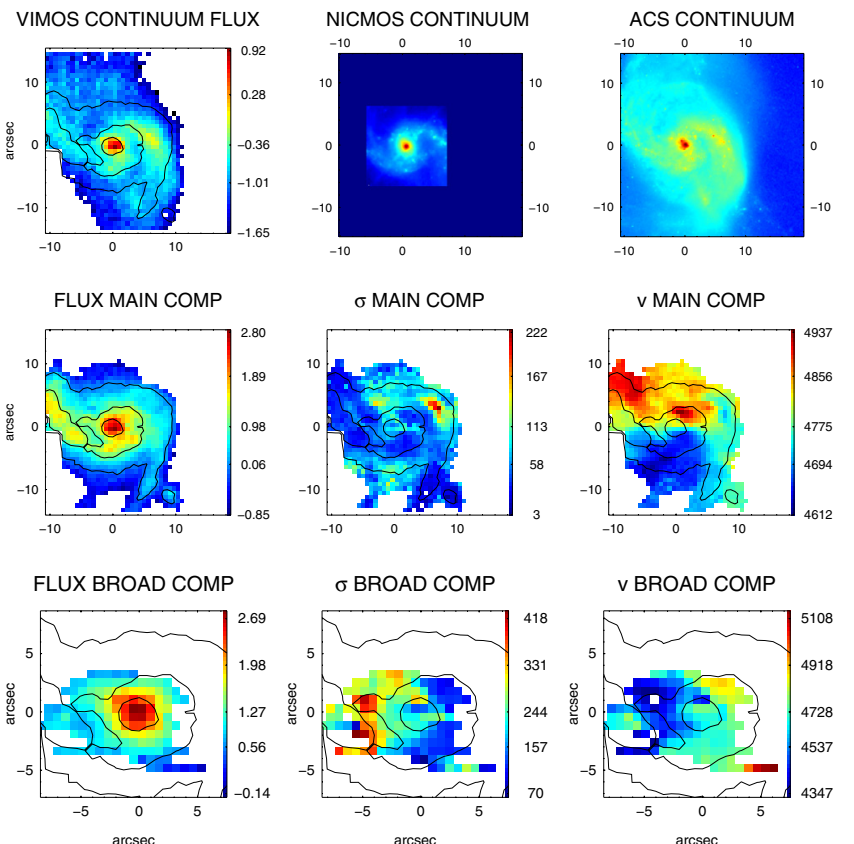

\section{$\sigma$ BROAD COMP}
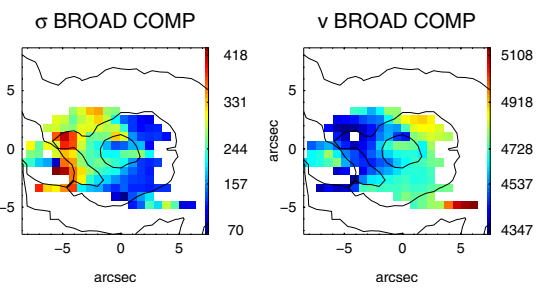

(0", 0")
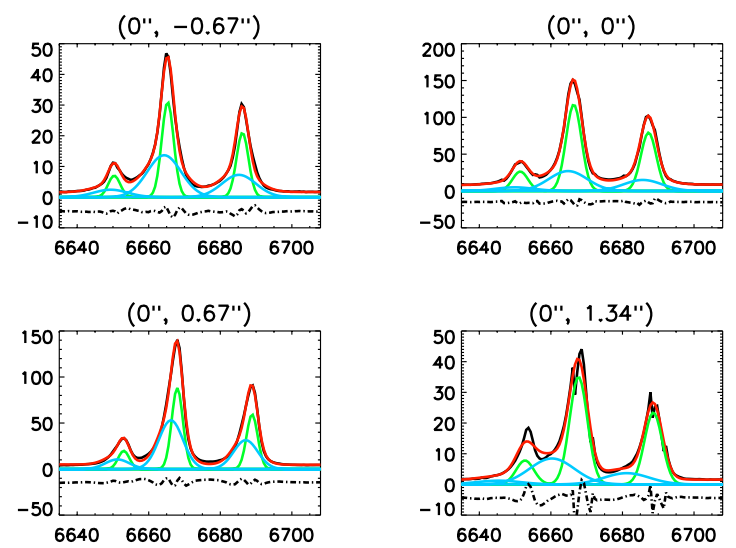

Fig. 3. Top panel: upper row: $\mathrm{H} \alpha$ maps for the class 2 object IRAS F04315-0840. VIMOS continuum image and HST continuum images (i.e., $H$ and $I$ bands). Middle row: flux intensity, velocity dispersion $\sigma\left(\mathrm{km} \mathrm{s}^{-1}\right)$, and velocity field $v$ maps $\left(\mathrm{km} \mathrm{s}^{-1}\right)$ for the main component. Lower row: respective maps for the broad component. Note the different FoV between the main and the broad components. The latter has been zoomed because it covers a small area. The flux intensity maps are represented on a logarithmic scale and in arbitrary flux units. All the images are centered using the $\mathrm{H} \alpha$ peak and the iso-contours of the $\mathrm{H} \alpha$ flux are overplotted. Bottom panel: $\mathrm{H} \alpha-[\mathrm{NII}]$ observed spectra of IRAS F04315-0840 for selected inner regions which are indicated in the top label using the same reference system as in the top panel where the main and broad components coexist. The red curve shows the total $\mathrm{H} \alpha$-[NII] components obtained from multi-component Gaussian fits. The green and blue curves represent, respectively, the main and broad components.

We have also used the kinematic information to derive the dynamical mass $M_{\text {dyn }}$. This mass takes into account the whole gravitational field, hence dark matter, gas, and stellar components are all included. Spiral galaxies can be modeled using a flat component for the disk and a spheroidal component for the central concentration (i.e., a de Vaucouleurs profile and a massive halo). Assuming that the central regions are virialized, the observed velocity dispersion of the ionized gas $\sigma_{\mathrm{c}}$ can provide a
Source F21453-3511
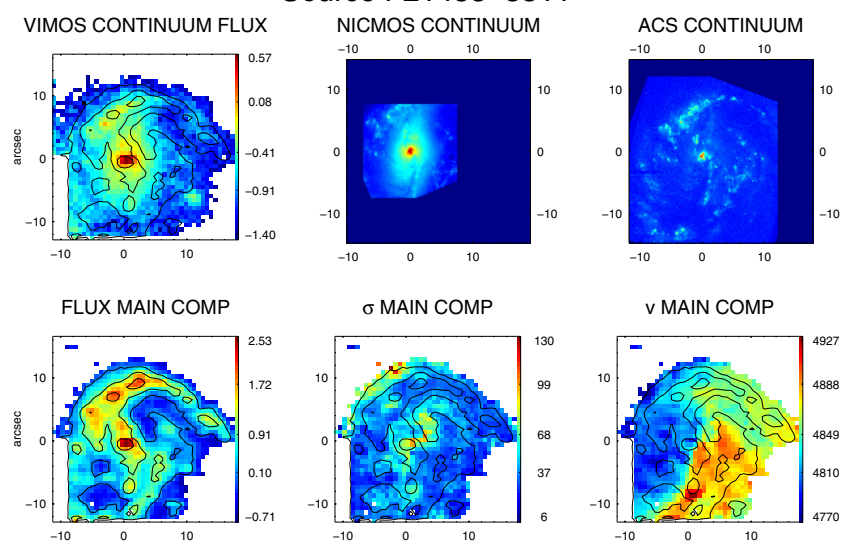

$\sigma$ MAIN COMP

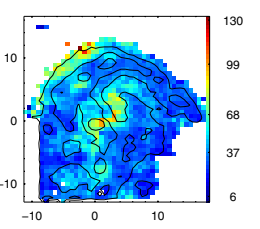

$\checkmark$ MAIN COMP

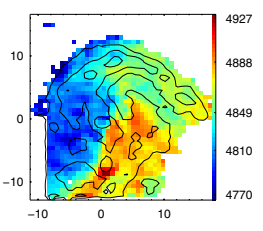

FLUX BROAD COMP

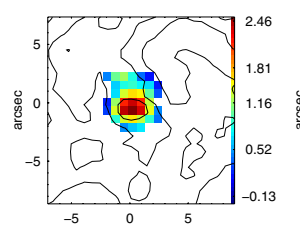

$\sigma$ BROAD COMP

v BROAD COMP
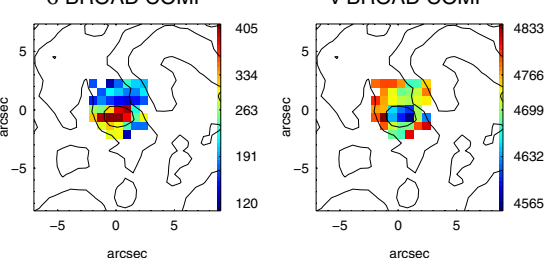

$\left(0 ",-1.34^{\prime \prime}\right)$

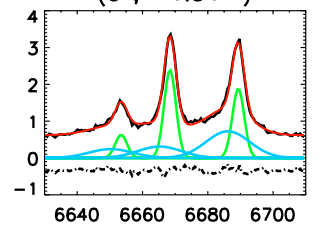

$\left(0^{\prime \prime},-0.67^{\prime \prime}\right)$
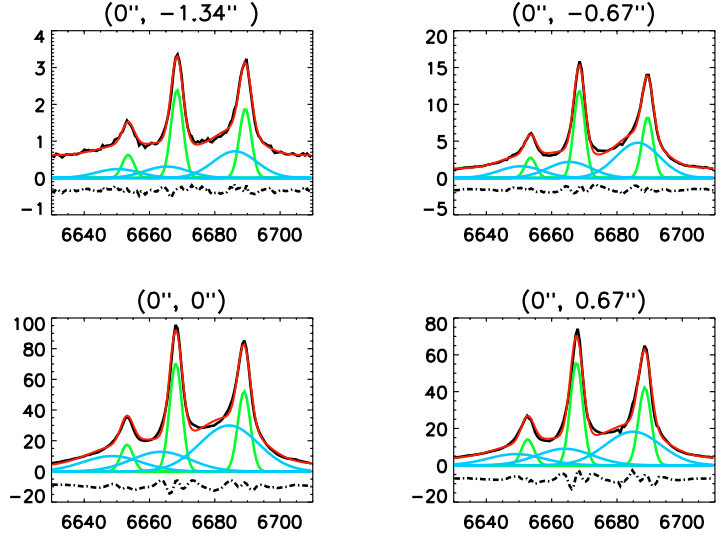

Fig.4. Top panel: upper row: $\mathrm{H} \alpha$ maps for the class 2 object IRAS F21453-3511. VIMOS continuum image and HST continuum images (i.e., $H$ and $I$ bands). Middle row: flux intensity, velocity dispersion $\sigma\left(\mathrm{km} \mathrm{s}^{-1}\right)$, and velocity field $v$ maps $\left(\mathrm{km} \mathrm{s}^{-1}\right)$ for the main component. Lower row: respective maps for the broad component. Note the different FoV between the main and the broad components. The latter has been zoomed in since it covers a small area. The flux intensity maps are represented on logarithmic scale and in arbitrary flux units. All the images are centered using the $\mathrm{H} \alpha$ peak and the iso-contours of the $\mathrm{H} \alpha$ flux are overplotted. Bottom panel: $\mathrm{H} \alpha$-[NII] observed spectra of IRAS F21453-3511 for a selected inner region (indicated by the coordinates in the top label using the same reference systems as in the top figure) where main and broad components coexist. The red curve shows the total $\mathrm{H} \alpha$-[NII] components obtained from multi-component Gaussian fits. The green and blue curves represent respectively the main and broad components.

reliable estimate of the disk's dynamical mass $\left(M_{\text {dyn }}\right.$, e.g., Colina et al. 2005). We use the relation:

$M_{\mathrm{dyn}}=m 10^{6} R_{\mathrm{hm}} \sigma_{\mathrm{c}}^{2} M_{\odot}$,

where $R_{\mathrm{hm}}$ is the half-mass radius in $\mathrm{kpc}$ and $\sigma_{\mathrm{c}}$ is the central velocity dispersion in $\mathrm{km} \mathrm{s}^{-1}$. The $R_{\mathrm{hm}}$ parameter is not an observable itself, hence cannot be measured directly, nor inferred by measuring the radius that encloses half luminosity of the galaxy (i.e., $\left.R_{\text {eff }}\right)$. The $R_{\text {eff }}$ were obtained from existing $2 \mathrm{MASS}$ 
Table 3. Kinematic parameters for the sample.

\begin{tabular}{lcccccccccc}
\hline \hline $\begin{array}{l}\text { Galaxy ID } \\
\text { IRAS code }\end{array}$ & Type of fitting $^{a}$ & $\begin{array}{c}i^{b} \\
\text { degree }\end{array}$ & $\begin{array}{c}v_{\mathrm{c}}{ }^{c} \\
\left(\mathrm{~km} \mathrm{~s}^{-1}\right)\end{array}$ & $\begin{array}{c}\sigma_{\mathrm{c}}{ }^{2} \\
\left(\mathrm{~km} \mathrm{~s}^{-1}\right)\end{array}$ & $v_{\mathrm{c}} / \sigma_{\mathrm{c}}$ & $\begin{array}{c}v_{\text {shear }}{ }^{e} \\
\left(\mathrm{~km} \mathrm{~s}^{-1}\right)\end{array}$ & $\begin{array}{c}\Sigma^{f} \\
\left(\mathrm{~km} \mathrm{~s}^{-1}\right)\end{array}$ & $v_{\text {shear }} / \Sigma$ & $\begin{array}{c}R_{\mathrm{eff}} \\
(\mathrm{kpc})\end{array}$ & $\begin{array}{c}M_{\mathrm{dyn}} \\
\left(M_{\odot}\right)\end{array}$ \\
\hline F11255-4120 & 1c & $53^{\circ} \pm 7^{\circ}$ & $187 \pm 34$ & $104 \pm 7$ & $1.8 \pm 0.5$ & & & & & \\
F11255-4120 & 2c & $53^{\circ} \pm 7^{\circ}$ & $188 \pm 37$ & $83 \pm 6$ & $2.3 \pm 0.6$ & 125 & 47 & 2.7 & $2.40 \pm 0.57$ & $(2.9 \pm 1.0) \times 10^{10}$ \\
F10567-4310 & $1 \mathrm{c}$ & $36^{\circ} \pm 4^{\circ}$ & $253 \pm 32$ & $56 \pm 3$ & $4.5 \pm 0.8$ & & & & & \\
F10567-4310 & 2c & $36^{\circ} \pm 4^{\circ}$ & $255 \pm 30$ & $50 \pm 2$ & $5.1 \pm 0.8$ & 120 & 41 & 3 & $3.20 \pm 0.40$ & $(1.4 \pm 0.3) \times 10^{10}$ \\
F04315-0840 & $1 \mathrm{c}$ & n.c. & $157 \pm 3$ & $110 \pm 11$ & $1.4 \pm 0.2$ & & & & & \\
F04315-0840 & 2c & n.c. & $162 \pm 3$ & $69 \pm 6$ & $2.4 \pm 0.3$ & 132 & 65 & 2 & $0.94 \pm 0.14$ & $(0.8 \pm 0.3) \times 10^{10}$ \\
F04315-0840 & $1 \mathrm{c}$ & $29^{\circ} \pm 3^{\circ}$ & $324 \pm 39$ & $110 \pm 11$ & $3.0 \pm 0.7$ & & & & & \\
F04315-0840 & 2c & $29^{\circ} \pm 3^{\circ}$ & $335 \pm 40$ & $69 \pm 6$ & $4.9 \pm 1.0$ & 132 & 65 & 2 & $0.94 \pm 0.14$ & $(0.8 \pm 0.3) \times 10^{10}$ \\
F21453-3511 & $1 \mathrm{c}$ & n.c. & $90 \pm 6$ & $69 \pm 8$ & $1.30 \pm 0.24$ & & & & & \\
F21453-3511 & 2c & n.c. & $78 \pm 5$ & $61 \pm 4$ & $1.28 \pm 0.17$ & 51 & 51 & $\sim 1$ & $2.97 \pm 0.82$ & $(1.9 \pm 0.8) \times 10^{10}$ \\
F21453-3511 & 1c & $24^{\circ} \pm 2^{\circ}$ & $222 \pm 33$ & $69 \pm 8$ & $3.2 \pm 0.9$ & & & & & \\
F21453-3511 & 2c & $24^{\circ} \pm 2^{\circ}$ & $192 \pm 28$ & $61 \pm 4$ & $3.2 \pm 0.7$ & 51 & 51 & $\sim 1$ & $2.97 \pm 0.82$ & $(1.9 \pm 0.8) \times 10^{10}$ \\
\hline
\end{tabular}

Notes. ${ }^{(a)}$ One-component fitting (1c) and two-component fitting (2c, referees to the main (systemic) component of the two-Gaussian fit) are considered for the four galaxies. ${ }^{(b)}$ Inclination of the galaxy; n.c. is when no inclination correction is applied. ${ }^{(c)}$ Circular velocity derived as the half of the observed peak-to-peak velocity from the $\mathrm{H} \alpha$ kinematic data. The errors are computed as the root mean square of the sum of the variances computed in an area of $4 \times 4$ spaxels around the two peaks (maximum and minimum) of the velocity field map. An additional mean error of 5-7 $\mathrm{km} \mathrm{s}^{-1}$ can be added when calibration errors are considered (see Table 2). Corrected and non-corrected values for the inclination are shown. ${ }^{(d)}$ Central velocity dispersion as derived from the $\sigma_{\mathrm{H} \alpha}$ maps. These errors are computed as done for the velocity field map. In this case, the calibration errors are on the order of 3-4 $\mathrm{km} \mathrm{s}^{-1} .{ }^{(e)}$ Velocity shear uncorrected for the inclination of the galaxy. ${ }^{(f)}$ Global velocity dispersion of the whole galaxy.

near-infrared imaging. The extinction in the near-IR is a factor of 5-10 lower than that in the optical and a proper way of minimizing these effects would be to measure the half-light radius in the $H$ or $K$ bands as done here. The parameter $m$ depends on the mass distribution: its value ranges from 1.4, for a King stellar mass distribution with a tidal-to-core ratio of 50, which is a good representation of elliptical galaxies (Tacconi et al. 2002), to 1.75 for a polytropic sphere with a density index covering a range of values (Spitzer 1987) and 2.1 for a de Vaucouleurs mass distribution (Combes et al. 1995). As in Colina et al. (2005), we assume $m=1.75$. All these results are shown in Table 3; their inclinations are drawn from the NED site and are consistent with our own estimates for the $\mathrm{H} \alpha$ distribution.

\subsection{IRAS F11255-4120 (ESO 319-G022)}

This is a class 0 object which appears to be a single isolated galaxy with a relatively symmetric morphology according to its DSS image. It is a barred spiral with a ring extending to $\sim 4 \mathrm{kpc}$ from the nuclear region that is clearly detected in our $\mathrm{H} \alpha$ image. The orientation of the bar is different in the VIMOS continuum image $\left(\mathrm{PA} \sim 110^{\circ}\right)$ and in the $\mathrm{H} \alpha$ image $\left(\mathrm{PA} \sim 150^{\circ}\right)$.

For the main component, as shown in the top panel of Fig. 1, the velocity dispersion $(\sigma)$ map has an almost centrally peaked structure with values of $120 \mathrm{~km} \mathrm{~s}^{-1}$ (high values also being found in the bar structure) and a lower mean value $\left(\sim 20 \mathrm{~km} \mathrm{~s}^{-1}\right)$ being found in the ring. The location of this peak does not agree with those of the $\mathrm{H} \alpha$ flux intensity and continuum maps with an offset of about $0.6 \mathrm{kpc}$ (i.e., $\sim 2$ arcsec). The kinematic center of the velocity field closely agrees with that of the $\mathrm{H} \alpha$ flux peak. The projected orientation of the rotation axis (minor kinematic axis) seems to be different by about $30^{\circ}$ from that of the bar in the $\mathrm{H} \alpha$ flux intensity map but is aligned with that of the bar in the continuum. The observed velocity amplitude for the main component at a distance of $4 \mathrm{kpc}$ from the $\mathrm{H}_{\alpha}$ peak is on the order of $(300 \pm 8) \mathrm{km} \mathrm{s}^{-1}$.
A small region of about $\sim 0.67 \mathrm{kpc} \times 0.89 \mathrm{kpc}$ in the nuclear region shows traces of a second component which is indicative of non-rotational mechanisms (e.g., outflow/wind). This component is blueshifted by $80 \mathrm{~km} \mathrm{~s}^{-1}$ with respect to the main one and has a large velocity dispersion $\sigma$ in the range (160-220) $\mathrm{km} \mathrm{s}^{-1}$ and a velocity amplitude on the order of $(60 \pm 7) \mathrm{km} \mathrm{s}^{-1}$.

The $v_{\mathrm{c}} / \sigma_{\mathrm{c}}$ and $v_{\text {shear }} / \Sigma$ have values of 2.3 and 2.7 , respectively, supporting the idea that this galaxy is rotationally supported: the kinematic properties of this galaxy seem to be consistent with its morphology (i.e., a rotating disk).

\subsection{IRAS F10567-4310 (ESO 264-G057)}

From the DSS image, IRAS F10567-4310 is morphologically classified as a class 0 object (see Paper III). The $\mathrm{H} \alpha$ flux map inferred from our IFU data (top panel of Fig. 2) shows a ring with a patchy distribution and its peak is in good positional agreement with the center of the galaxy as inferred from the continuum.

The main component shows a centrally peaked velocity dispersion map with typical values of $60-70 \mathrm{~km} \mathrm{~s}^{-1}$. Its general morphology resembles that of the $\mathrm{H} \alpha$ flux map (i.e., regions with high $\mathrm{H} \alpha$ surface brightness tend to have large velocity dispersions, with a mean value of $40 \mathrm{~km} \mathrm{~s}^{-1}$ ). This component has a disk-like regular velocity field with a clear rotation pattern, where the kinematic center in good positional agreement with the $\mathrm{H} \alpha$ flux peak. The observed velocity amplitude is about $(300 \pm 6) \mathrm{km} \mathrm{s}^{-1}$ when computed at a distance of $4.7 \mathrm{kpc}$ from the kinematic center.

A secondary kinematically distinct component is identified in the nuclear region over an area of $\sim 1.2 \mathrm{kpc} \times 0.9 \mathrm{kpc}$; it has a velocity amplitude of $(128 \pm 9) \mathrm{km} \mathrm{s}^{-1}$ and is blue-shifted by $\sim 70 \mathrm{~km} \mathrm{~s}^{-1}$ with respect to the main one; its velocity dispersion is in the range $(90-180) \mathrm{km} \mathrm{s}^{-1}$.

The $v_{\mathrm{c}} / \sigma_{\mathrm{c}}$ parameter clearly classifies this object as rotationdominated with $v_{\mathrm{c}} / \sigma_{\mathrm{c}} \sim 5$ and $v_{\text {shear }} / \Sigma \sim 3$. The kinematics of this class 0 object are also consistent with its morphology. 


\subsection{IRAS F04315-0840 (NGC 1614)}

This is a class 2 object (Fig. 3) with a relatively asymmetric morphology: the DSS image shows a tidal tail extending for $13 \mathrm{kpc}$ from the nuclear region. This is a well-studied, post-coalescence late merger, with a bright, spiral structure of a length scale of few kpc $(1-3 \mathrm{kpc})$. The spiral structure in the $\mathrm{H} \alpha$ flux map has a different orientation from the continuum.

The velocity dispersion map of the main component has an irregular structure. Its peak (i.e., $220 \mathrm{~km} \mathrm{~s}^{-1}$ ) is found at $2.4 \mathrm{kpc}$ from the nucleus (i.e., $\mathrm{H} \alpha$ flux peak) in the northern arm. The velocity field of the main component is somewhat distorted and chaotic with an amplitude of $(325 \pm 5) \mathrm{km} \mathrm{s}^{-1}$. The projection of the rotation axis in the outer part (connecting the north-east to south-west part) is not aligned with that of the inner part, which is aligned as north-south direction.

A broad component is found in the inner region and covers a relatively large area of $\sim 2.4 \mathrm{kpc} \times 2.7 \mathrm{kpc}$. This component is blue-shifted by $50 \mathrm{~km} \mathrm{~s}^{-1}$ with respect to the main component. Its velocity dispersion is in the range (70-400) $\mathrm{km} \mathrm{s}^{-1}$ with a velocity amplitude of $(760 \pm 13) \mathrm{km} \mathrm{s}^{-1}$. The projections of the kinematic major axes of the main and broad component differ by $90^{\circ}$ and the blue-shifted region of the velocity field of the broad component has the largest velocity dispersion (i.e., $400 \mathrm{~km} \mathrm{~s}^{-1}$ ), supporting the hypothesis of a dusty outflow, where the receding components (which are behind the disk) are obscured, making the whole profile relatively narrow with respect to the approaching component.

We derive a $v_{\text {shear }} / \Sigma \sim 2$ and a $v_{\mathrm{c}} / \sigma_{\mathrm{c}}>1$ even when the inclination correction is not included (i.e., $v_{\mathrm{c}} / \sigma_{\mathrm{c}} \approx 1.4-2.4$ ): this illustrates the importance of intrinsic rotation over random motion. Therefore, this parameter implies that we should classify this object as rotation-dominated.

\subsection{IRAS F21453-3511 (NGC 7130)}

IRAS F21453-3511 is a peculiar class 2 object, with traces of spiral and asymmetric morphology from the HST and DSS images. The ionized gas, as traced by the $\mathrm{H} \alpha$ emission, is concentrated in the nuclear region and mostly in the northern spiral arm.

The asymmetric velocity dispersion map of the main component clearly has higher values in the northern arm and its central part (i.e., two local maxima can be revealed) with values of $80 \mathrm{~km} \mathrm{~s}^{-1}$. The velocity field of the same component is asymmetric, and three main regions can be identified: the south-west (SW) part has a mean value of $4900 \mathrm{~km} \mathrm{~s}^{-1}$, the north-west (NW) one with $4850 \mathrm{~km} \mathrm{~s}^{-1}$ and the eastern (E) part has $4780 \mathrm{~km} \mathrm{~s}^{-1}$. The NW part could have a different inclination from the remaining observed regions, which can explain this peculiar velocity structure. The kinematic center can be clearly identified with the $\mathrm{H}_{\alpha}$ flux peak, although the rotational axis is not well-defined. The photometric major axis in the NICMOS image seems to be orientated in the NS direction and the ACS image seems to reveal a ring of knots with a major axis orientated along PA $\sim 135^{\circ}$.

A small region of about $1.5 \mathrm{kpc} \times 1.3 \mathrm{kpc}$ in the nuclear part contains a second component. The blue-shifted region of its velocity field has the largest velocity dispersion (i.e., $\sigma \sim$ $400 \mathrm{~km} \mathrm{~s}^{-1}$, see Fig. 4). This feature suggests that an outflow is present in the inner part of the galaxy. The amplitude of the velocity dispersion map is about $(285 \pm 20) \mathrm{km} \mathrm{s}^{-1}$, while the velocity field amplitude is $(270 \pm 16) \mathrm{km} \mathrm{s}^{-1}$. This component is blue-shifted by $150 \mathrm{~km} \mathrm{~s}^{-1}$ with respect to the main component.

The derived $v_{\mathrm{c}} / \sigma_{\mathrm{c}}$ and $v_{\text {shear }} / \Sigma$ parameters are respectively closed to 1.3 (when no inclination correction is included) and 1: in this object, the random motions do not seem to dominate, although in its velocity field and velocity dispersion maps there are some anomalies. On the basis of these parameters, we would classify this source as rotation dominated.

\subsection{Summary of the global kinematical properties}

From the kinematic maps and the results derived so far (see Table 3), we can draw some general conclusions. Class 0 objects are characterized by point-antisymmetric velocity fields, showing the spider diagram that is typical of a ideal rotating disk; their velocity dispersion maps are centrally peaked with a radial decay at larger radii. On the other hand, class 2 objects have velocity fields that do not follow this pattern. Despite being to first approximation point-antisymmetric, they also display clear disturbances and asymmetries, which could be explained by a bent disk and/or other tidal motions, consequences of the past merger. Their $\sigma$ maps are more asymmetric with off-nuclear regions of peculiarly large velocity dispersion values (e.g., IRAS F043150840), and asymmetrical structures in the nuclear regions (e.g., IRAS F21453-3511).

All of our sources contain broad and blue-shifted components (i.e., with velocity shifts of $\Delta v=(50-150) \mathrm{km} \mathrm{s}^{-1}$ with respect to the main component) in the inner parts (nucleus) of the galaxies. They have mean velocity dispersions of (140-260) $\mathrm{km} \mathrm{s}^{-1}$, which are 2-4 times larger than those of the main components. These components cover a small area in disk objects (i.e., $\sim 1 \mathrm{kpc}^{2}$ ), while larger areas (i.e., 2-6 $\mathrm{kpc}^{2}$ ) are involved for the post-coalescence mergers, which display more complex and distorted spectra. Although this component is more prominent for the class 2 objects, it is blueshifted for the four sources and has high associated velocities suggests that an outflow can exist in their inner regions. Furthermore, the velocity field patterns of this component (i.e., kinematic axes perpendicular to those of the main component) support the dusty outflow hypothesis.

From our kinematic results, we see that the sole use of one-dimensional (1D) parameters $v_{\mathrm{c}} / \sigma_{\mathrm{c}}$ and/or $v_{\text {shear }} / \Sigma$ does not seem to give us a unique classification for our sources. According to them, our objects indeed seem to be rotation dominated, even when the inclination correction is not included. Their $v_{\mathrm{c}} / \sigma_{\mathrm{c}}$ values are in-between those typical of local spiral galaxies (i.e., $v_{\mathrm{c}} / \sigma_{\mathrm{c}} \sim 5-15$, Epinat et al. 2010) and Lyman break analogs at $z \sim 0.2$ (with $v_{\mathrm{c}} / \sigma_{\mathrm{c}} \sim 0.4-1.8$, Gonçalves et al. 2010).

The derived global velocity dispersions $\Sigma$ are generally (40-60) $\mathrm{km} \mathrm{s}^{-1}$, which is comparable to those obtained in Gonçalves et al. (2010) for LBAs, with a typical median value of $\sim 67 \mathrm{~km} \mathrm{~s}^{-1}$, but much higher than those observed in other lower luminosity local star-forming galaxies (i.e., typical $\sigma \sim$ 5-15 $\mathrm{km} \mathrm{s}^{-1}$, e.g., Dib et al. 2006). For comparison, we also derived the $v_{\text {shear }}$ (not corrected for the inclination of the galaxy) as explained before. Our values range between $50 \mathrm{~km} \mathrm{~s}^{-1}$ (i.e., IRAS F04315-0840) and $130 \mathrm{~km} \mathrm{~s}^{-1}$. This parameter reveals higher degree of rotation than the values obtained for LBAs (i.e., $v_{\text {shear }}<70 \mathrm{~km} \mathrm{~s}^{-1}$ ), where in many cases a significant velocity gradient was not observed, and no actual rotation can be identified. We then derive values of $v_{\text {shear }} / \Sigma$ typical of rotation dominated objects (i.e., $\sim 2-3$, Epinat et al. 2010) with the exception of the galaxy IRAS F04315-0840 (see Table 3).

The derived dynamical masses classify our LIRGs as moderate mass systems, characterized by a mean value of $(1.8 \pm$ $0.4) \times 10^{10} M_{\odot}$. Our mass estimates agree with those obtained for LIRGs in Hinz \& Rieke (2006) and Väisänen et al. (2008), which are in the range $\left(10^{10}-10^{11}\right) M_{\odot}$. 
The post-coalescence mergers do not have extreme kinematic asymmetries in their maps, making them ideal targets to explore the potential of kinemetry for characterizing moderate asymmetries.

\section{Kinemetry analysis}

We investigate the potential of the kinemetry method developed by Krajnović et al. (2006, hereafter K06), in analyzing the kinematic maps of (U)LIRGs. In particular, we want to investigate the power of this methodology in studying the kinematic asymmetries. First we apply the kinemetry method to the sample, drawing preliminary conclusions about the kinematics of these objects. We then will apply the same criteria as those proposed by Shapiro et al. (2008) and also explore the potential of a new criterion to study kinematic asymmetries. We therefore expect to measure lower asymmetries for our two post-coalescence merger systems than to those considered by S08, which are more kinematically disturbed because of their recent merger activity. In our post-coalescence systems, the inner parts are expected to be relaxed into an almost virialized disk with a large rotational component, while the outer parts should still retain asymmetries associated with the merger events. Finally, we analyze the resolution and redshift effects on these results.

\subsection{The method}

The kinemetry method, developed by Krajnović et al. (2006), comprises a decomposition of the moment maps into Fourier components using ellipses. For clarity, we briefly describe again the main steps presented in K06 to achieve a clearer understanding of this analysis. The Fourier analysis is the most straightforward way to characterize any periodic phenomenon: the periodicity of a kinematic moment can easily be seen by expressing the moment in polar coordinates where $K(x, y) \rightarrow K(r, \psi)$. The map $K(r, \psi)$ can be expanded as follows to a finite number $(N+1)$ of harmonic terms (frequencies)

$K(r, \psi)=A_{0}(r)+\sum_{n=1}^{N} A_{n}(r) \sin (n \cdot \psi)+B_{n}(r) \cos (n \cdot \psi)$,

where $\psi$ is the azimuthal angle in the plane of the galaxy (measured from the major axis) and $r$ is the radius of a generic ellipse. The harmonic series can be presented in a more compact way as

$K(r, \psi)=A_{0}(r)+\sum_{n=1}^{N} k_{n}(r) \cdot \cos \left[n\left(\psi-\phi_{n}(r)\right)\right]$,

where the amplitude and the phase coefficients $\left(k_{n}, \phi_{n}\right)$ are easily calculated from the $A_{n}, B_{n}$ coefficients as $k_{n}=\sqrt{A_{n}^{2}+B_{n}^{2}}$ and $\phi_{n}=\arctan \left(\frac{A_{n}}{B_{n}}\right)$. Thus, for an ideal rotating disk one would expect the velocity profile to be dominated by the $B_{1}$ term, while the velocity dispersion profile dominated by the $A_{0}$ term. In the case of an odd moment ( $\left.\mu_{\text {odd }}\right)$, the sampling ellipse parameters are determined by requiring that the profile along the ellipse is well-described by $\mu_{\text {odd }}(\psi, r) \approx B_{1}(r) \cdot \cos (\psi)$ since the velocity field peaks at the galaxy major axis $(\psi=0)$ and goes to zero along the minor axis $(\psi=\pi / 2)$. Hence, the power in the $B_{1, v}$ term therefore represents the circular velocity in each ring $r$, while power in other coefficients represents deviations from circular motion. On the other hand, the zeroth-order term, $A_{0}$, gives the systemic velocity of each ring. The velocity dispersion field is an even moment $\left(\mu_{\text {even }}\right)$ of the velocity distribution, so that its kinematic analysis is identical to traditional surface photometry. In an ideal rotating disk, the velocity dispersion has to be constant along each ring of the ellipse $\left(\mu_{\text {even }}(\psi, r) \approx A_{0}(r)\right)$ and decreases when the semi-major axis length increases. In this context, the $A_{0}$ term represents the velocity dispersion profile. Higher order terms $\left(A_{n}, B_{n}\right)$ indicate deviations from symmetry (Krajnović et al. 2006).

There are two effects that limit the reliability with which coefficients in the expansion can be determined: (i) the absolute number of points sampled along the ellipse, and (ii) the regularity with which these points sample the ellipse as a function of angle, $\theta$. Ellipse parameters (i.e., position angle $\Gamma$, centre, and flattening $q=b / a$ ) can be determined by minimizing a small number of harmonic terms; in the code, some of them can be constrained (e.g., fixing the center allows the position angle $\Gamma$ and flattening $q$ to freely change or remain fixed). The choice of a correct center is important in the analysis in order to avoid artificial overestimations of the asymmetries.

\subsection{Kinemetry of LIRGs sample}

In our analysis, the position of the galaxy's center is assumed to correspond the peak of the $\mathrm{H} \alpha$ flux intensity map. In the two class 0 objects, the $\mathrm{H} \alpha$ peak is in good positional agreement with the center of symmetry of the velocity field (i.e. kinematic center). For the class 2 objects, the kinematic center of the velocity field is not so well-defined, but the $\mathrm{H} \alpha$ peak is also in a reasonable symmetric position. The position angle $\Gamma$ is allowed to vary. The flattening $q$ is also allowed to vary, but across a physically meaningful range (i.e. 0.2-1). This allows us to consider general cases, such as tilted/wrapped disks. In Sect. 4.3.1. (i.e., Fig. 10), we discuss further the effects of the different choices on these input parameters.

In Figs. 5-8, we present for the four galaxies the original kinematic maps (i.e., velocity field and velocity dispersion), along with their reconstructed maps (i.e., obtained using all the coefficients of the harmonic expansion up to the fifth order corrections). The residual maps (i.e., original - model) are also shown with a typical rms of $10 \mathrm{~km} \mathrm{~s}^{-1}$. Therefore, in all four cases, the fits are good and the reconstructed maps recover the properties of the original data in great detail. We also present the behavior of the kinematic parameters defined in the previous section (i.e., position angle, $\Gamma$, and the flattening, $q$ ), along with some of the kinemetry coefficients $\left(k_{1}\right.$ and $k_{5}$ normalized to the $k_{1}$ ).

As for the ellipse parameters, we find in general a good optimization for $\Gamma$ at the different radii for the four objects. As for $q$, there are several cases that reach the boundary of the range of acceptable values. In general, this happens in the innermost regions where the rotation component, and therefore the amplitude of the sinusoidal velocity profile along the ellipse, are small. The irregularities in the velocity field are therefore far larger than the (rotational) amplitude of the velocity profile. Since the problem of finding the best-fit ellipse (i.e., best-fit $q$ and $\Gamma$ combination) can be quite degenerate with many local minima, a low $q$ (high ellipticity) facilitates the minimization of $\chi^{2}$ because the latter is sensitive to a change in $\Gamma$. In contrast, if $q$ is high (circular ellipse), the fit $\left(\chi^{2}\right)$ is insensitive to a change in $\Gamma$ increasing the difficulty of the $\chi^{2}$ minimization process. As a result, $q$ tends to have small values in the inner regions, reaching in some cases the minimum acceptable value. In addition, the $q$ parameter is more weakly constrained at smaller radii as a consequence of the relatively fewer data points involved and the 


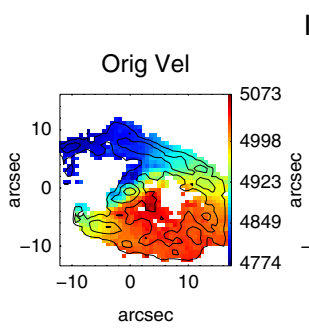

IRAS $11255-4120$

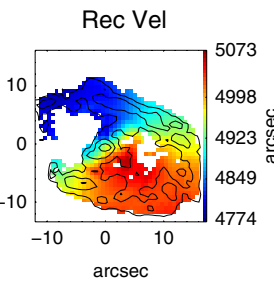

arcsec

Orig Vel Disp

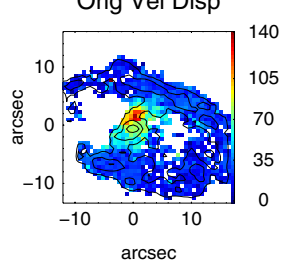

Rec Vel Disp
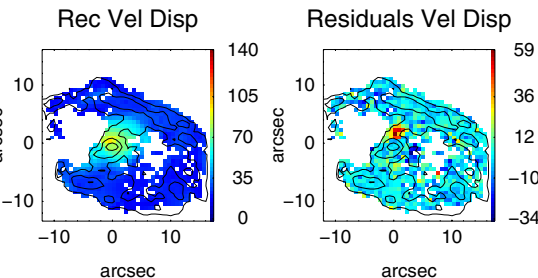

IRAS F11255-4120 ( $Q, \Gamma$ free, COVER $=0.7,10$ params)

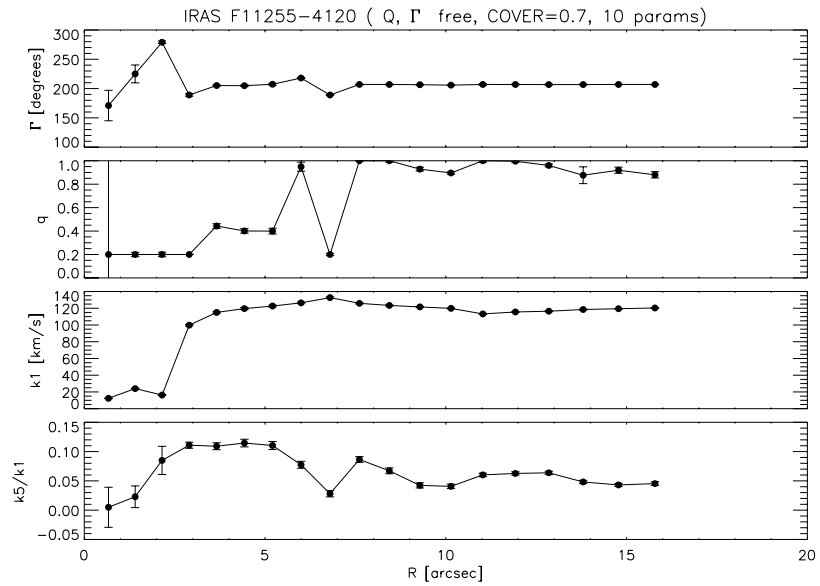

Fig. 5. Upper panel: maps of $\mathrm{H} \alpha$ Gaussian fit velocities (top left), $\mathrm{H} \alpha$ Gaussian fit dispersion (bottom left) and their respective reconstructed (middle) and residual (data-model) maps (on the right) for IRAS F11255-4120. Lower panel: radial profiles of the kinematic properties, obtained using kinemetry program. The position angle $\Gamma$ and the flattening $q$ of the best-fit ellipses, as well as the first and the fifth order Fourier terms (respectively, $k_{1}$ and $k_{5}$ ) are plotted as a function of the radius.

seeing smearing, and has larger errors ${ }^{3}$. This does not mean, however, that the harmonic expansion to describe the kinematics is not well-constrained. The reconstructed velocity maps are indeed in excellent agreement with the real velocity field for the inner regions (i.e., low $q$ values), even for the innermost region (see Figs. 5-8). At larger $r$, the amplitude of the velocity profile along the ellipse is larger; more data points contribute to constrain the ellipse, that are less sensitive to local kinematic irregularities, hence provide a more optimal fitting of the parameters. At large radii, we also find good agreement between the fit and the data.

We now provide some comments on the kinemetry results for each galaxy of the sample.

- IRAS F11255-4120. The position angle $\Gamma$ is quite stable $\left(\sim 200^{\circ} \pm 15\right)$ over most the sampled radius. In the inner part (i.e. $r<5^{\prime \prime}$ ), $q$ has values of $0.2-0.4$ and it has some

\footnotetext{
3 Note that kinemetry provides the errors in the ellipse parameters (i.e., $q, \Gamma)$, which are determined by the Levenberg-Marquardt least squares minimization (MPFIT) fit with the formal $1 \sigma$ uncertainties computed from the covariance matrix. Similarly, the harmonic terms have their formal $1 \sigma$ errors estimated from the diagonal elements of the corresponding covariance matrix obtained with a linear least squares fit.
}
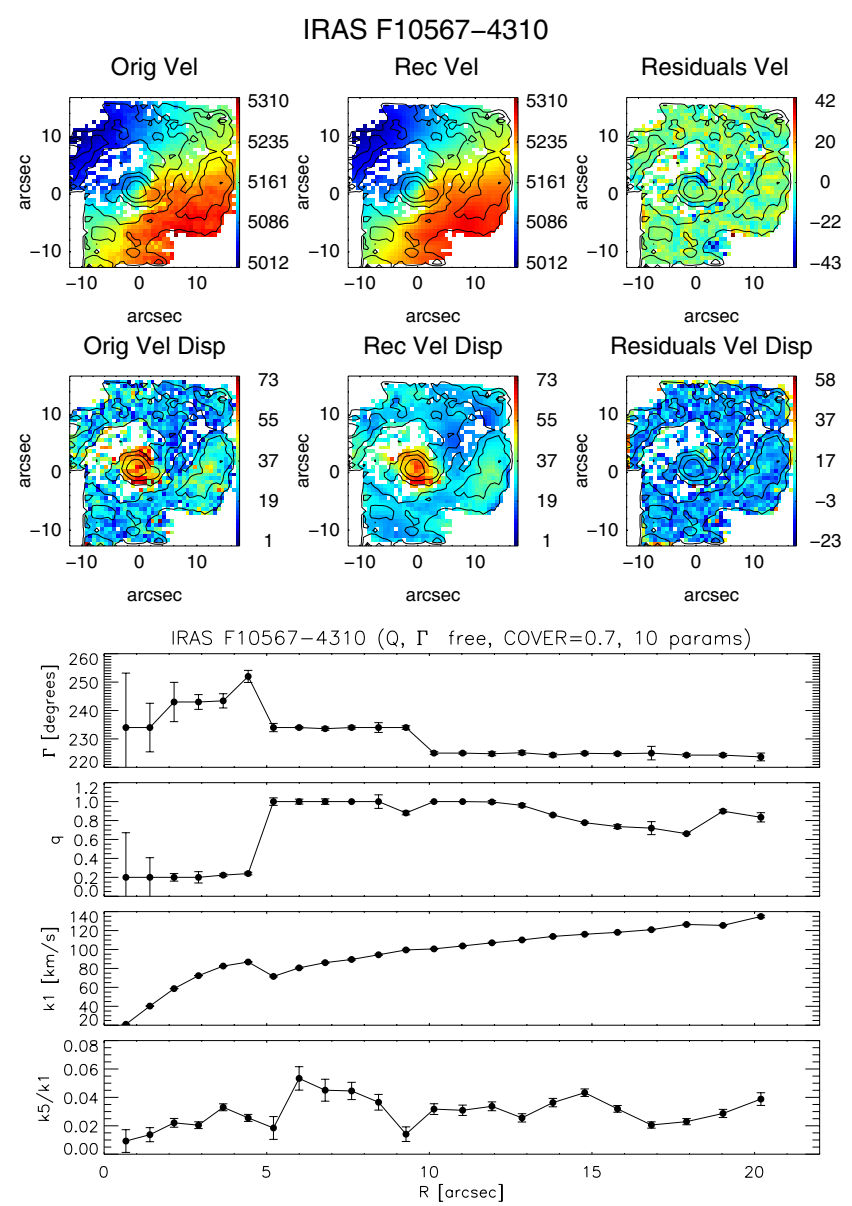

Fig. 6. Upper panel: maps of $\mathrm{H} \alpha$ Gaussian fit velocities (top left), $\mathrm{H} \alpha$ Gaussian fit dispersion (bottom left) and their respective reconstructed (middle) and residual (data-model) maps (on the right) for IRAS F10567-4310. Lower panel: radial profiles of the kinematic properties, obtained using kinemetry program. The position angle $\Gamma$ and the flattening $q$ of the best-fit ellipses, as well as the first and the fifth order Fourier terms (respectively, $k_{1}$ and $k_{5}$ ) are plotted as a function of the radius.

instabilities at $r \sim 7^{\prime \prime}$, which are likely due to the inner bar structure. However, the expansion recovers very well the data in all this region. We find that $k_{1}$ is mainly dominated by rotation (i.e., $B_{1}$ ) and increases radially up to a value of $140 \mathrm{~km} \mathrm{~s}^{-1}$. The $k_{5} / k_{1}$ term, which measures the small-scale kinematic asymmetries, has small values up to a maximum of 0.1 , which is indicative of minor peculiarities for $r<7^{\prime \prime}$, likely due to the bar structure. These asymmetries become smaller in the outer parts. The error bars are not visible because they are smaller than the black points in the plot.

- IRAS F10567-4310. The position angle $\Gamma$ is quite stable over the sampled radii, with values between 210 and 250 degrees. The $q$ parameter reaches the value of 0.2 in the inner region (i.e., $r<4^{\prime \prime}$ ), where the reconstructed map shows a very good agreement with the data. The parameter $k_{1}$ is mainly dominated by rotation (i.e., $B_{1}$ ) and increases radially up to a value of $140 \mathrm{~km} \mathrm{~s}^{-1}$, revealing a possible minor anomaly at $r \sim 5^{\prime \prime}$. The $k_{5} / k_{1}$ term has very small values, with a maximum of 0.05 over the sampled radii, meaning that this object is close to being an ideal rotating disk structure. 


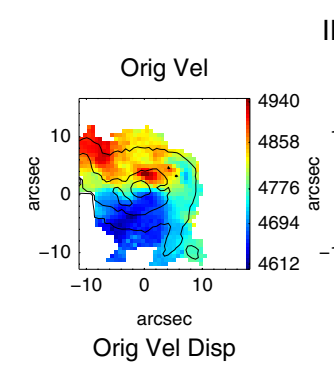

IRAS 04315-0840

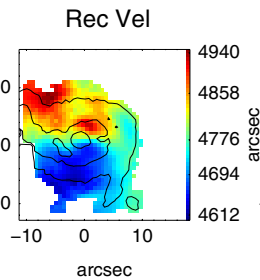

Rec Vel Disp

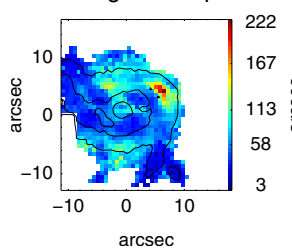

$\operatorname{arcsec}$

IRAS F04315-0840 ( $Q, \Gamma$ free, COVER $=0.7,10$ params $)$

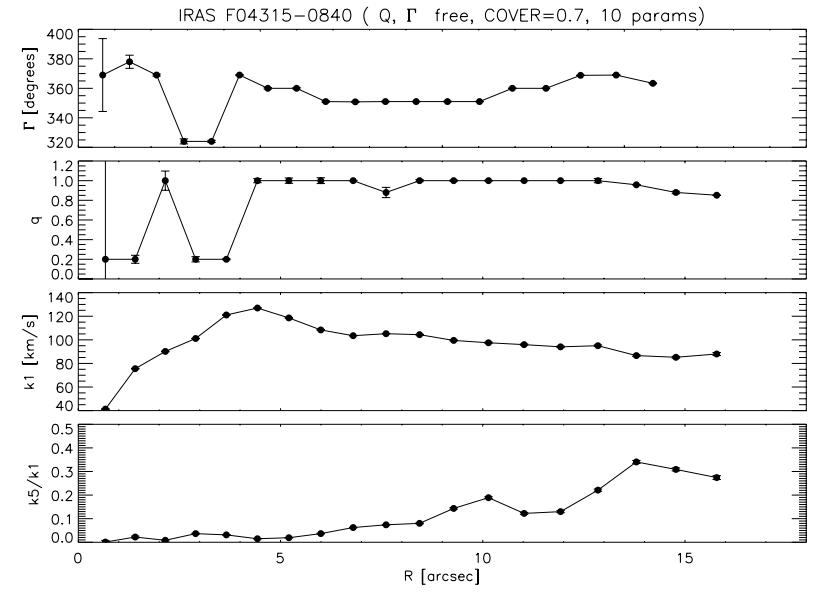

Fig. 7. Upper panel: maps of $\mathrm{H} \alpha$ Gaussian fit velocities (top left), $\mathrm{H} \alpha$ Gaussian fit dispersion (bottom left) and their respective reconstructed (middle) and residual (data-model) maps (on the right) for IRAS F04315-0840. Lower panel: radial profiles of the kinematic properties, obtained using kinemetry program. The position angle $\Gamma$ and the flattening $q$ of the best-fit ellipses, as well as the first and the fifth order Fourier terms (respectively, $k_{1}$ and $k_{5}$ ) are plotted as a function of the radius.

- IRAS F04315-0840. The position angle $\Gamma$ typically spans from $350^{\circ}$ to $380^{\circ}$, except for $\left(r \sim 3^{\prime \prime}\right)$, which drops to around $320^{\circ}$ as a consequence of one of two distinct redshifted peaks present in the velocity field map. For most of the radii, $q$ is close to 1 (circle-ellipses). The parameter $k_{1}$ increases radially up to a value of $120 \mathrm{~km} \mathrm{~s}^{-1}\left(r<5^{\prime \prime}\right)$ and then decreases to reach a value of $100 \mathrm{~km} \mathrm{~s}^{-1}$ at $r \sim 15^{\prime \prime}$. The $k_{5} / k_{1}$ term is between 0 and 0.3 , and has a constant increasing behavior. This illustrates that departures from rotation are mainly found in the outer parts of the galaxy.

- IRAS F21453-3511. The position angle $\Gamma$ remains almost constant to $\sim 240^{\circ}$ for $r>6^{\prime \prime}$, while it has lower and more unstable values for $r<6^{\prime \prime}$. The values of $q$ reaches 0.2 in the region within a radius of $r<6^{\prime \prime}$, mainly owing to the relatively small rotation component in this region. However, the agreement between the kinematic fit and data is very good for these radii. At $r=7^{\prime \prime}, q$ reaches a maximum $(\sim 1)$ and then decreases constantly to a value of 0.4 at larger radii.

\footnotetext{
4 The position angle is measured from north $\left(0^{\circ}=360^{\circ}\right)$ anticlockwise. To ensure that the two values become more easily comparable, we choose to add $360^{\circ}$ in one case.
}

IRAS 21453-3511

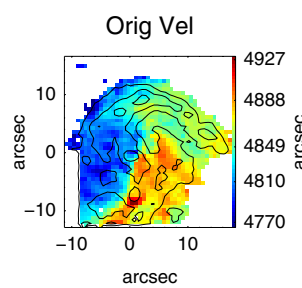

arcsec
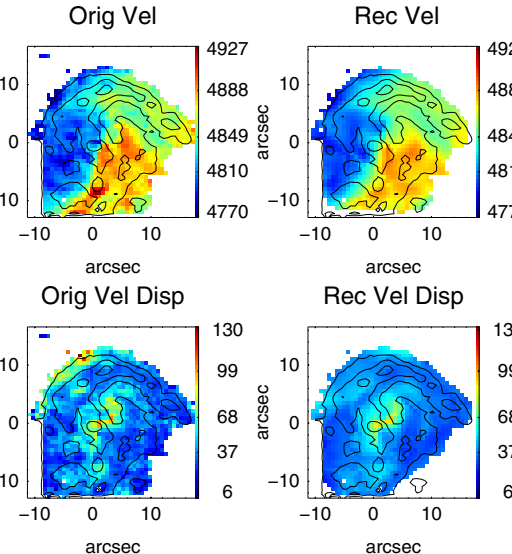

Rec Vel Disp

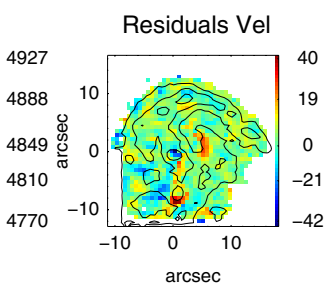

Residuals Vel Disp
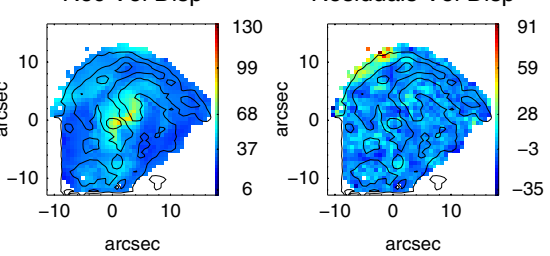

IRAS F21453-3511 (Q, $\Gamma$ free, COVER $=0.7,10$ params)
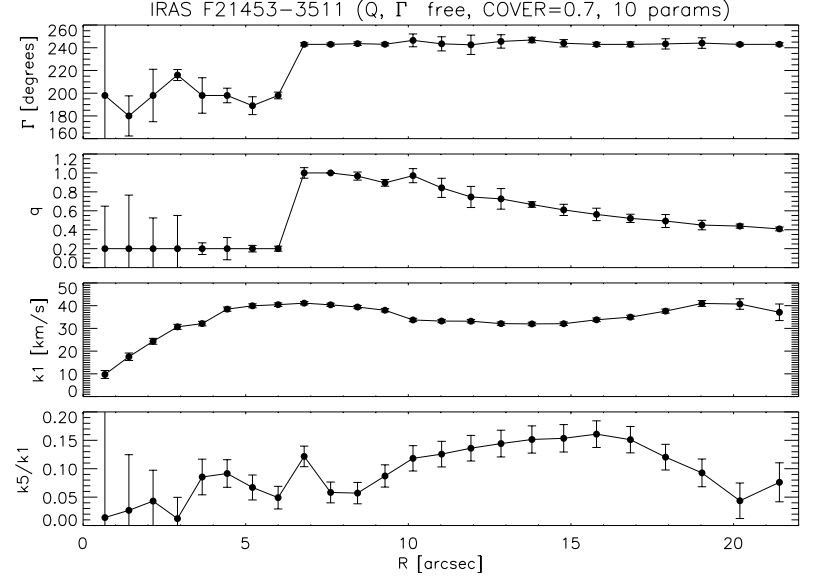

Fig. 8. Upper panel: maps of $\mathrm{H} \alpha$ Gaussian fit velocities (top left), $\mathrm{H} \alpha$ Gaussian fit dispersion (bottom left) and their respective reconstructed (middle) and residual (data-model) maps (on the right) for IRAS F21453-3511. Lower panel: radial profiles of the kinematic properties, obtained using kinemetry program. The position angle $\Gamma$ and the flattening $q$ of the best-fit ellipses, as well as the first and the fifth order Fourier terms (respectively, $k_{1}$ and $k_{5}$ ) are plotted as a function of the radius.

$k_{1}$ does not reveal any strong rotational component ranging between $10 \mathrm{~km} \mathrm{~s}^{-1}$ and $40 \mathrm{~km} \mathrm{~s}^{-1}$. In the inner region it increases for $r<4^{\prime \prime}$ and then remains constant. The $k_{5} / k_{1}$ term ranges between 0 and 0.2 , showing a quite stable trend. For radii larger than $10^{\prime \prime}$, it follows the opposite behavior of the $k_{1}$ term. In this object, the deviations from pure rotation are not as large as those of IRAS F04315-0840, but they are larger than those for the class 0 objects.

In general, we have found that for class 0 sources the higher order deviations (from pure rotation) are small (i.e., $k_{5} / k_{1}<0.1$ ), while for class 2 objects the deviations are larger, particularly in the outer regions $\left(k_{5} / k_{1} \leq 0.4\right)$. For all of these sources, the rotation curve (i.e., $k_{1}$ parameter) seems to imply that these objects are rotating. As discussed before, these galaxies do not display extreme asymmetries in their kinematic maps as confirmed by the kinemetry results obtained so far.

In the following sections, we study several kinematic criteria to enhance our classification of these systems.

\subsection{Sample of local LIRG systems in the $\left[\sigma_{a}-v_{a}\right]$ plane}

To reveal rotational/non-rotational motion within the dynamics of the gas in each galaxy, we first consider the same criteria 
E. Bellocchi et al.: Kinematic asymmetries of disks and post-coalescence mergers

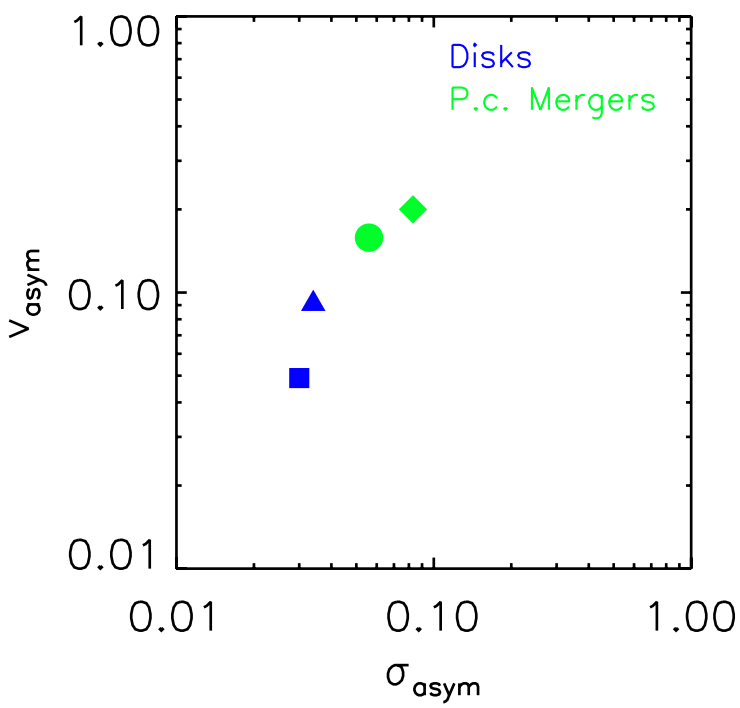

Fig. 9. Asymmetry measure of the velocity $v_{\text {asym }}$ and velocity dispersion $\sigma_{\text {asym }}$ fields for the four galaxies analyzed here. Symbol types distinguish the different systems: the square represents IRAS F10567-4310, the triangle is IRAS F11255-4120, the circle is IRAS F04315-0840, and the diamond represents IRAS F21453-3511. Blue and green symbols represent different morphological types, respectively class 0 and class 2 objects.

as the one proposed by S08. We note that they compare two main classes of systems: those that have suffered a recent major merger event (i.e., mergers) and those without any signs of interacting or merger activity (i.e., disks). For further details, we refer to S08. They define the asymmetries in the velocity and velocity dispersion fields as

$v_{\mathrm{asym}}=\left\langle\frac{k_{\mathrm{avg}, v}}{B_{1, v}}\right\rangle_{r} \quad \sigma_{\mathrm{asym}}=\left\langle\frac{k_{\mathrm{avg}, \sigma}}{B_{1, v}}\right\rangle_{r}$,

where $k_{\mathrm{avg}, v}=\left(k_{2, v}+k_{3, v}+k_{4, v}+k_{5, v}\right) / 4$ and $k_{\mathrm{avg}, \sigma}=\left(k_{1, \sigma}+\right.$ $\left.k_{2, \sigma}+k_{3, \sigma}+k_{4, \sigma}+k_{5, \sigma}\right) / 5$. For an ideal rotating disk, we expect the velocity profile to be perfectly antisymmetric if the $B_{1}$ term dominates the Fourier expansion, while the velocity dispersion map is expected to be perfectly symmetric and therefore all terms except $A_{0}$ should vanish.

In Fig. 9, we show the results for our galaxies in the $\left[\sigma_{a}-v_{a}\right]$ plane. As expected, class 0 objects have lower values of the asymmetries than class 2 objects. Looking at their velocity and velocity dispersion maps, the class 0 maps (Figs. 5, 6) resemble those of an ideal rotating disk (i.e., "spider diagram" structure for the velocity field and centrally peaked velocity dispersion map), while class 2 objects have more distorted velocity fields and irregular dispersion maps (Figs. 7, 8). Therefore, taking into account that the objects in the present sample were classified as either class 0 or class 2 based on pure morphological arguments, we can conclude that their morphological and kinematical classification in the $\left[\sigma_{a}-v_{a}\right]$ plane are consistent.

To analyze the robustness of the results in Fig. 9, we now analyze their dependence on the considered input parameters as well as the errors in the radial velocities and velocity dispersion measurements. We note that kinemetry uses the full 2D kinematic information of the velocity field and velocity dispersion map, allowing us to perform a good characterization of the asymmetries, something that is more efficient than using 1D parameters such as $v_{\mathrm{c}} / \sigma_{\mathrm{c}}$ and $v_{\text {shear }} / \Sigma$ as seen in Sect. 3.

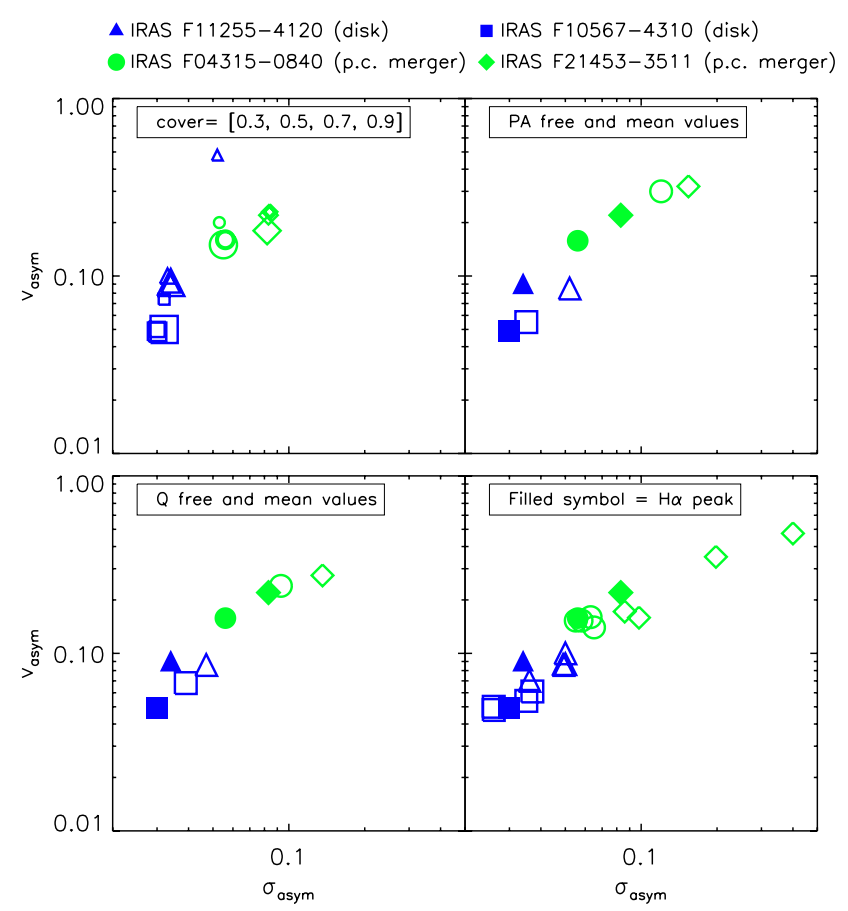

Fig. 10. Results for the asymmetry measures when different sets of input parameters are considered with a ten harmonic term analysis. Symbol types and colors help us to distinguish among the different systems as explained in the legend. In each panel, one parameter at a time is changed, namely respectively, COVER, $\Gamma$ (or PA), $q$, and the CENTER of the ellipses. Top-left: different results for the COVER parameter (i.e., $0.3,0.5,0.7,0.9)$ where the size of the symbol is proportional to the COVER value (the biggest symbol corresponds to the highest cover value and vice versa). Top-right: the results obtained when we allow a position angle $\Gamma$ to vary (filled symbols) or be fixed to its mean value (empty symbols) are shown. Bottom-left: the results shown are obtained when we allow $q$ to either vary or remain constant with radius at its mean value. Bottom-right: results achieved choosing five different centers for each galaxy: filled symbols represent results obtained from the "standard" analysis (H $\alpha$ flux peak), and empty symbols the results obtained by shifting their center by one pixel (horizontally and vertically, with respect to the $\mathrm{H} \alpha$ peak pixel corresponding to a shift of $0.95^{\prime \prime}$ ).

\subsubsection{Dependence on the input parameters}

As pointed out by K06, a good choice of input parameters is important to avoid an artificial overestimation of the asymmetries. To perform a kinemetry analysis, we need to define the dynamical center, as well as specify different levels of constraints of the input parameters. For instance, the kinematic position angle $\Gamma$ and the flattening $q$ of the ellipses can be fixed or allowed to vary in the fitting at different radii. The COVER parameter, which controls the radius at which the process stops by setting the fraction of the ellipse that has to be covered by data ${ }^{5}$, also has to be assigned.

In Fig. 10, we present the results in the $\left[\sigma_{a}-v_{a}\right]$ plane of our galaxies for different sets of input parameters. In each panel, one parameter at a time is changed, respectively, the COVER (top-left), the position angle $\Gamma$ (or PA, top-right), the flattening $q$ (bottom-left), and the CENTER (bottom-right) of the ellipses. We can find that the results are stable for a reasonable

5 For instance, COVER $=0.7$ means that if fewer than $70 \%$ of the points along an ellipse are not covered by data the program stops. This value ensures sure that kinematic coefficients are robust. It is sometimes necessary to relax this condition, especially when reconstructing maps. For further details, we refer to Krajnović et al. (2006). 
choice of values. When considering the COVER panel, the four galaxies give similar results up to $\operatorname{cover}=0.5$, whereas IRAS F11255-4120 deviates significantly for COVER = 0.3. Our results for IRAS F21453-3511 seem to be sensitive to our choice of galaxy center but it remains stable within the region of highasymmetries in all the (extreme) cases considered. As shown, the choice of either free or fixed position angle $\Gamma$ or flattening $q$ do not have a strong effect on the final results. In particular, the computed asymmetries are quite insensitive to the choice for $q$, especially for the class 0 galaxies. In general, the asymmetries obtained for a fixed $\Gamma / q$ are somewhat higher because there is smaller degree of freedom such that the asymmetries cannot be accurately taken into account in the fitting. The proper choice of free/fixed parameters obviously depends on the level of $\mathrm{S} / \mathrm{N}$ (i.e., if the deviations are associated with a true feature or noise). In accordance with these results, we selected the input parameters for our analysis of COVER $=0.7$, where $\Gamma$ is completely allowed to vary, $q$ is free to vary in the range $[0.2-1]$, and the center $=$ H $\alpha$ flux peak.

\subsubsection{Monte Carlo simulations}

To analyze the dependence of the kinemetry results on the uncertainties in the radial velocities and velocity dispersion values, we measure the probability distribution functions (PDFs) of the asymmetries in these systems using Monte Carlo (MC) simulations, as done in S08, since the kinemetry method does not lend itself to a straightforward error propagation.

Therefore, for each template, we create 150 different realizations of the moment maps (i.e., velocity field and velocity dispersion) based on their corresponding error maps. These error maps correspond to the measurement errors in the velocity moments, as derived when fitting the kinematics in the data cube along with wavelength calibration errors, as explained in Sect. 2.4. For each moment map, we perturb the observed data points by randomizing them, using Gaussian noise parameterized by the measured $1 \sigma$ errors. The new maps created are then used to rerun kinemetry and apply the analysis described before. In Fig. 11, our results are shown. We find that our results in the $\left[\sigma_{a}-v_{a}\right]$ plane are almost unaffected by the velocity errors and that there are relatively well-defined regions for disks and merger galaxies.

\subsection{Total kinematic asymmetry of disk, ongoing, and post-coalescence merger systems}

As done in S08, we compute the total kinematic asymmetry $K_{\text {tot }}$ border in order to separate class 0 (disks) and class 2 (postcoalescence merger) objects. For the four objects, we obtained a mean value of $\left\langle K_{\text {tot }}\right\rangle=0.135$ which is significantly lower value than that obtained in S08 (i.e., $\left\langle K_{\text {tot }}\right\rangle=0.5$ ). The difference could arise because our mergers are in a post-coalescence phase displaying relaxation in the innermost regions with a large rotation component, while in S08 they are mostly pre-coalescence merger systems dominated by irregular velocity field and high velocity dispersion. In addiction, the limit defined in S08 comes out after excluding IRAS 12112+0305, a pre-coalescence merger pair (i.e., García-Marín et al. 2009) which resembles a disk at high redshift. If the classification of this object had considered, the total kinematic asymmetry border derived for the whole S08 sample would have been considerably lower (i.e., $\left\langle K_{\text {tot }}\right\rangle \sim 0.3$, see Fig. 5 in S08), hence the discrepancy from our findings may be smaller.
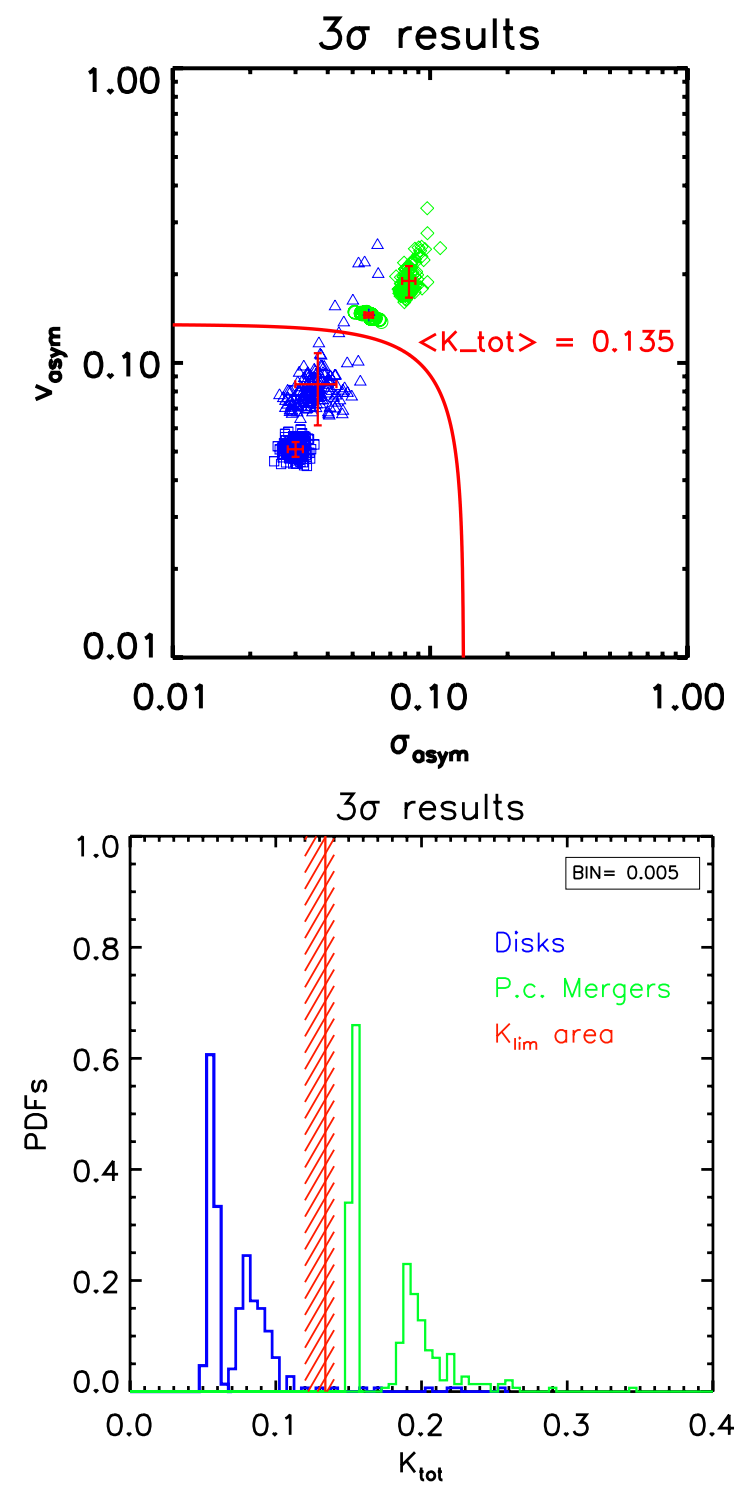

Fig. 11. Top: asymmetry measures of the velocity $v_{\text {asym }}$ and velocity dispersion $\sigma_{\text {asym }}$ fields derived from the Monte Carlo realizations for the four objects. For each source, $150 \mathrm{MC}$ simulations are run but here only the $\pm 3 \sigma$ results are shown. The solid red line indicates the division between class 0 (disks) and class 2 (post-coalescence mergers) at $\left\langle K_{\text {tot }}\right\rangle=0.135$. Bottom: the probability distribution function (PDFs) as derived from $\mathrm{MC}$ realizations. The empirical delineation where $K_{\text {lim }}=0.135$ can cleanly separate the two classes (i.e., $K_{\text {lim }}$ area). The symbols are the same as those used before.

In any case, the border disk/merger for the S08 sample is so dependent on the classification of a single object (i.e., IRAS $12112+0305)$ illustrates its relatively large associated uncertainties. A reduction in the total asymmetry border $K_{\text {tot }}$, as our results (and S08, after reclassifying IRAS 12112+0305) suggest, may also lead to a change in the relative frequency of disks and mergers in a given sample, hence an increase in the fraction of mergers. Changing the relative frequencies of disks and mergers has obvious implications for the interpretation of our data in terms of the different evolutionary scenarios mentioned in the introduction. On the other hand, our sample is admittedly too small and consists of objects with relatively homogeneous properties (i.e., LIRGs classified as disks and post-coalescence mergers) for a robust determination of general use. Therefore, additional 
efforts to constrain its value as well as understand how it depends on several instrumental and observational factors are required.

\subsection{A new criterion to distinguish kinematic asymmetries between post-coalescence mergers from disks}

To better assess the presence of asymmetries in the kinematic maps, we explore a new kinematic criterion.

As described in Kronberger et al. (2007), when considering recent (i.e., $\leq 100 \mathrm{Myr}$ after the first encounter) or ongoing major mergers of equal mass galaxies (i.e., Milky Way type), the inner regions ${ }^{6}$ of the galaxy are usually affected by more chaotic motions, as revealed by a quite irregular rotational curve and higher order deviations (i.e., $k_{5} / k_{1}$ ) at small radii. As the major merger evolves, the inner regions rapidly relax into a rotating disk, while the outer parts remain out of equilibrium. This implies that the velocity field in a post-coalescence system might be dominated by rotation in the inner regions but have large kinematic asymmetries in the outer parts, as is actually observed in our systems. Provided that the outer regions retain better the memory of a merger event, we propose a criterion that enhances the relative importance of the asymmetries at larger radii.

Instead of simply averaging the asymmetries over all radii (as in S08), we then weight these according to the number of data points used in their determination. Since the number of data points of the outer ellipses is larger than for the inner ones, the asymmetries found in the outer ellipses contribute more significantly to the average when deriving $v_{\text {asym }}$ and $\sigma_{\text {asym }}$. As the number of data points is to first approximation proportional to the circumference of the ellipse, for practical reasons we used this to weight the asymmetries found for the different ellipses.

The circumferences of the ellipses are computed using the truncated "infinite sum" formula, which is a function of the ellipticity (i.e., $e=\sqrt{1-q^{2}}$ ) and the semi-major axis of the ellipse $(r)$

$C(e, r) \approx 2 \pi r\left[1-\left(\frac{1}{2}\right)^{2} e^{2}-\left(\frac{1 \cdot 3}{2 \cdot 4}\right)^{2} \cdot \frac{e^{4}}{3}\right]$.

The final formula to compute the weighted velocity and velocity dispersion asymmetries are respectively

$v_{\mathrm{asym}}=\sum_{n=1}^{N}\left(\frac{k_{\mathrm{avg}, n}^{v}}{B_{1, n}^{v}} \cdot C_{n}\right) \cdot \frac{1}{\sum_{n=1}^{N} C_{n}}$,

$\sigma_{\mathrm{asym}}=\sum_{n=1}^{N}\left(\frac{k_{\mathrm{avg}, n}^{\sigma}}{B_{1, n}^{v}} \cdot C_{n}\right) \cdot \frac{1}{\sum_{n=1}^{N} C_{n}}$,

where $N$ is the total number of radii considered, $C_{n}$ the value of the circumference for a given ellipse, the different $k_{n}\left(k_{n}^{v}\right.$ and $\left.k_{n}^{\sigma}\right)$ are the deviations concerning respectively the velocity field and velocity dispersion maps, and $B_{1}^{v}$ is the rotational curves. We refer to this approach as the "weighted" method and its associated plane $\left.\mathrm{W}-\left[\sigma_{a}-v_{a}\right]\right)$. In Fig. 12, the results in the $\mathrm{W}-\left[\sigma_{a}-v_{a}\right]$ plane for our four galaxies are shown, where MC were performed as described above (Sect. 4.3.2).

The results follow the same general trend but the two classes are distinguished somewhat more clearly than in the unweighted

\footnotetext{
${ }^{6}$ If a galaxy covers a FoV of $30 \mathrm{kpc} \times 30 \mathrm{kpc}$, the regions more affected by chaotic motions are those at galactocentric distances smaller than $10 \mathrm{kpc}$.
}
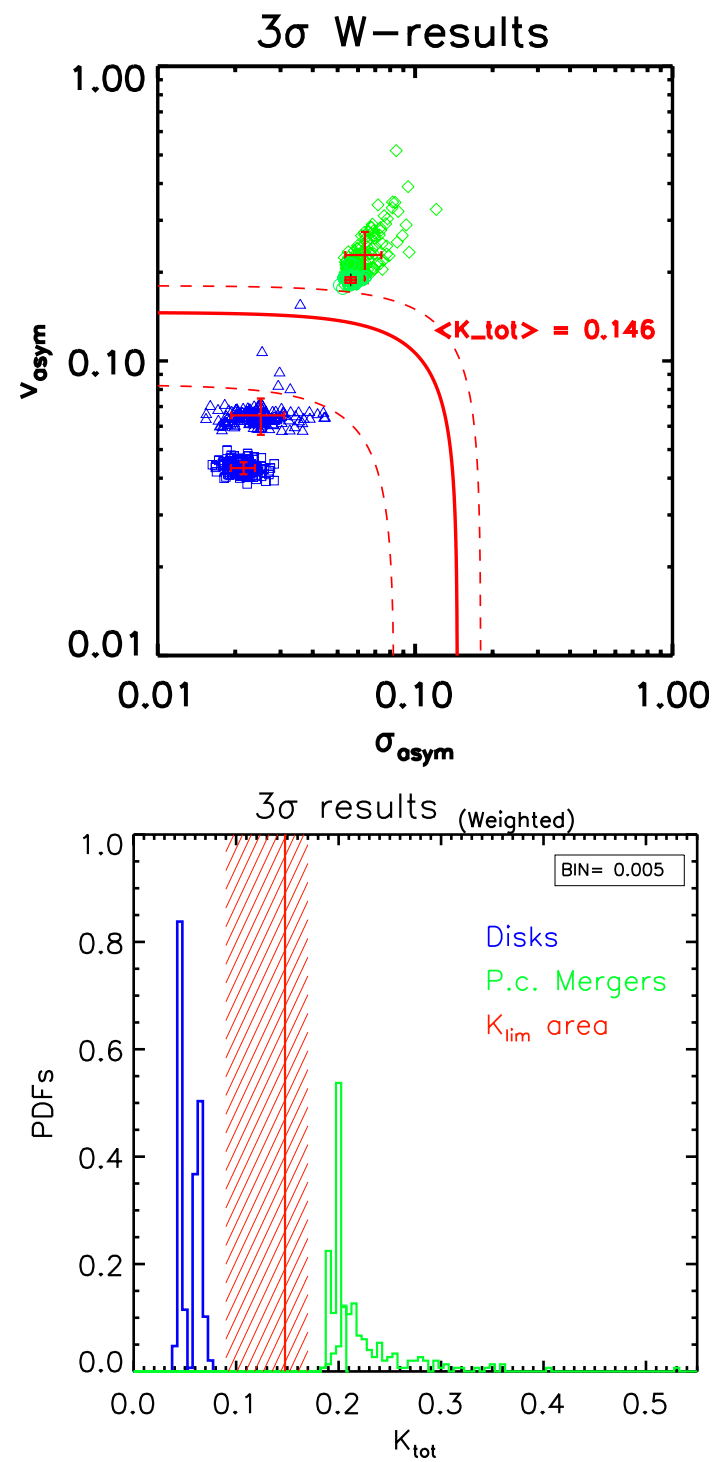

Fig. 12. Top: weighted asymmetry measures of the velocity $v_{\text {asym }}^{(w)}$ and velocity dispersion $\sigma_{\text {asym }}^{(w)}$ fields derived from the Monte Carlo realizations for the four objects. For each source, $150 \mathrm{MC}$ simulations are run but only the $\pm 3 \sigma$ results are shown. The solid red line indicates the empirical division between disks and post-coalescence mergers at $\left\langle K_{\text {tot }}\right\rangle=0.146$. Bottom: the probability distribution function (PDFs) as derived from MC realizations. The empirical delineation $K_{\lim }=0.146$ clearly distinguishes the two classes along with a large range of other values (i.e., dashed $K_{\lim }$ area).

case (Sect. 4.3). For class 0 objects, the weighted velocity asymmetries are lower, while for class 2 objects these asymmetries are somewhat higher than in the $\left[\sigma_{a}-v_{a}\right]$ plane. Therefore, this highlights that post-coalescence mergers have larger deviations at larger radii with respect to pure rotational motions, while disks have still smaller deviations than those obtained using $\left[\sigma_{a}-v_{a}\right]$. In this case, the total kinematic asymmetry border, which distinguishes the two disks and the two post-coalescence mergers, is characterized by a mean value of 0.146 .

\subsection{Angular resolution/redshift dependence}

The effects of angular resolution on the distortions of the velocity fields produced by mergers were discussed by Kronberger et al. (2007) based on simulated velocity fields as a function of 


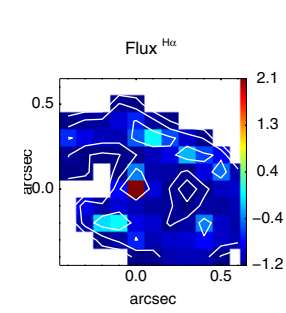

IRAS F11255-4120
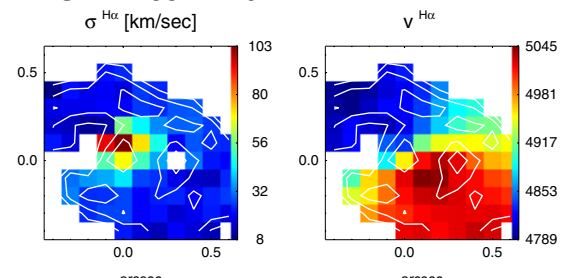

IRAS F10567-4310
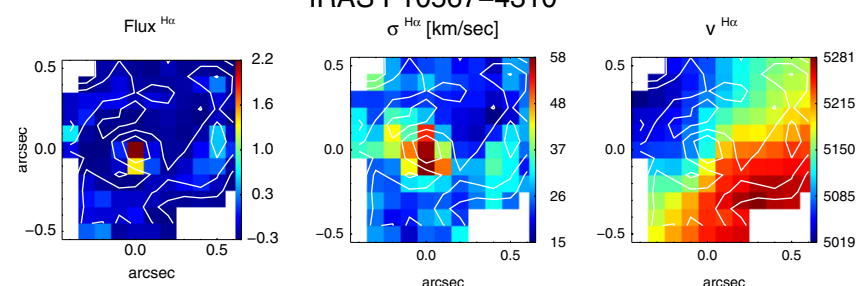

IRAS F04315-0840

Flux ${ }^{\text {Ho }}$
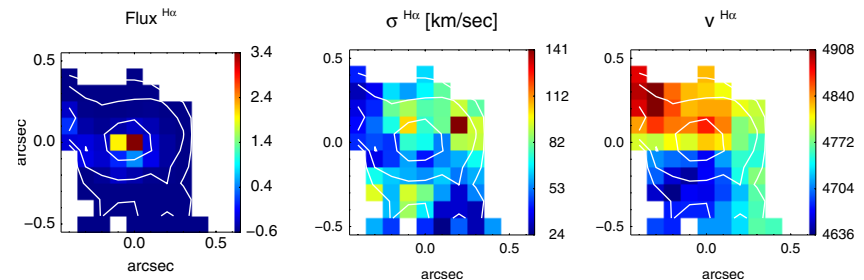

IRAS F21453-3511
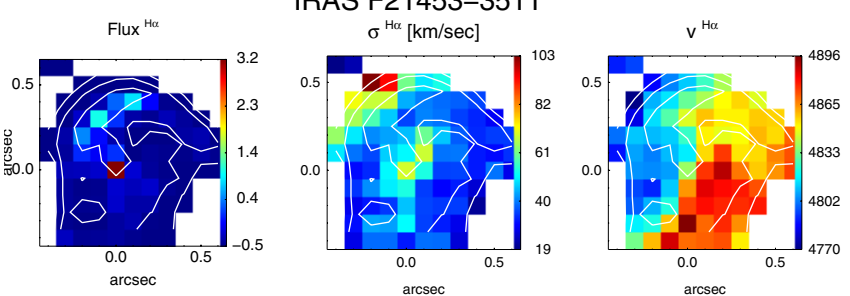

Fig. 13. $\mathrm{H} \alpha$ maps for the four objects observed at $z=3$ with a spatial sampling of $0.1^{\prime \prime}$. The flux intensity, velocity dispersion $\sigma\left(\mathrm{km} \mathrm{s}^{-1}\right)$, and velocity fields $v$ maps $\left(\mathrm{km} \mathrm{s}^{-1}\right)$ for the main component are shown. The flux intensity maps are represented on a logarithmic scale and in arbitrary flux units. All the images are centered using the $\mathrm{H} \alpha$ peak and the iso-contours of the $\mathrm{H} \alpha$ flux are over-plotted.

redshift (i.e., $0<z<1$ ). They found that for large (Milky Way type) galaxies the distortions are clearly visible at intermediate redshifts but partially smeared out, while for small galaxies even strong distortions cannot be seen in the velocity field at $z \approx 0.5$.

Gonçalves et al. (2010), simulating LBAs at redshift $z \sim 2$, found that, in general, galaxies at high redshift have smaller values of $K_{\text {asym }}$, i.e., they appear more disky than they actually are. The percentage of galaxies classified as mergers drop from $\sim 70 \%$ to $\sim 38 \%$ from $z=0$ to $z=3$ according to their simulations.

To investigate the effects of resolution on our results, we simulate the observation of these systems at $z=3$ with a typical pixel scale of $0.1^{\prime \prime}$ (the same pixel scale as the IFU NIRSpec/JWST). At this redshift, the current FoV of our images is about $1^{\prime \prime} \times 1^{\prime \prime}$ with a typical scale of $7.83 \mathrm{kpc} / \mathrm{arcsec}$, assuming a $\Lambda$ CDM cosmology with $H_{0}=70 \mathrm{~km} \mathrm{~s}^{-1} \mathrm{Mpc}^{-1}, \Omega_{\mathrm{M}}=0.3$, and $\Omega_{\Lambda}=0.7$. The simulated maps are shown in Fig. 13. We apply kinemetry using these maps and, following the same procedures as before, obtain the results shown in the $\left[\sigma_{a}-v_{a}\right]$ (Fig. 14) and $\mathrm{W}-\left[\sigma_{a}-v_{a}\right]$ (Fig. 15) planes. The $\left[\sigma_{a}-v_{a}\right]$ plane shows the expected trend, where both classes appear to be more symmetric when observed at high redshift. In this case, a lower value of the

\section{Unweighted Low/High-z}

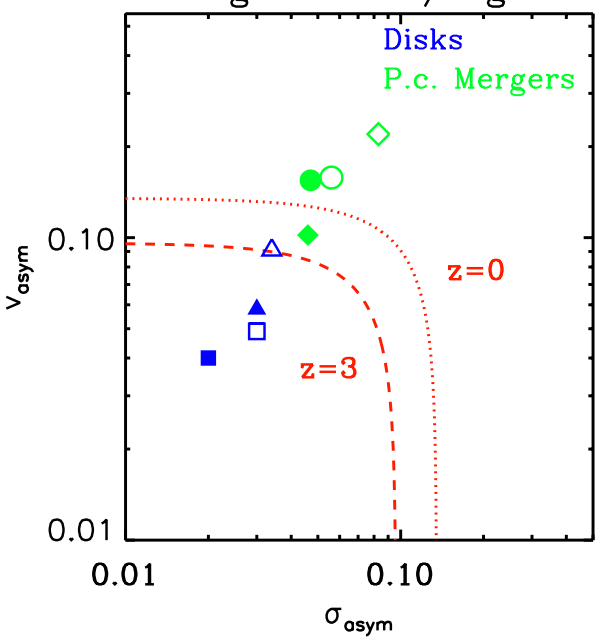

Fig. 14. The comparison between local and $z=3$ results in the $\left[\sigma_{a}-\right.$ $v_{a}$ ] plane. Empty symbols represent low- $z$ results, while filled ones are for $z=3$. Symbols have the same meaning as in Fig. 9. The red dashed line is the high-z frontier with a value of 0.096 as explained in the text while the dotted one is for the local analysis (i.e., 0.135).

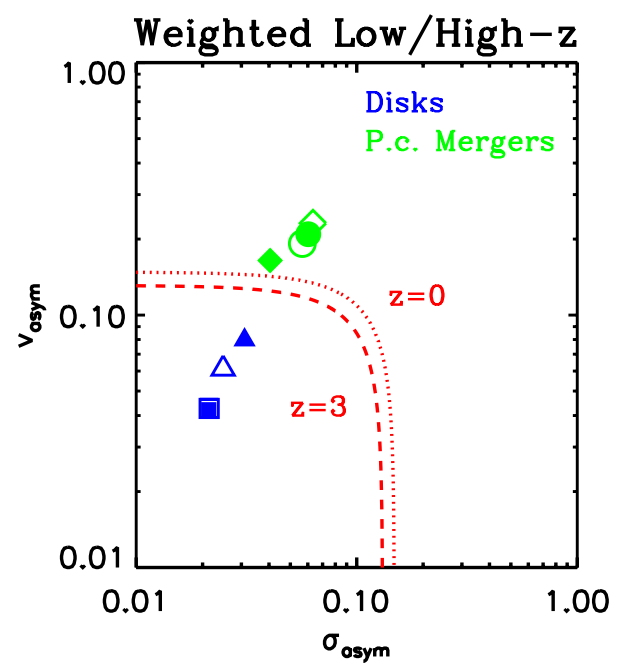

Fig. 15. Comparison between local and $z=3$ results in the W- $\left[\sigma_{a}-\right.$ $v_{a}$ ] plane. Empty symbols represent low- $z$ results, while filled ones are for $z=3$. The red dashed line represents the high-z frontier, while the dotted one is for the local case.

total kinematic asymmetry $K_{\text {asym }}$ border is derived (red dashed line), as expected (i.e., $K_{\text {asym }}=0.096$ ). Thus, shifting the sample from $z=0$ to $z=3$, the frontier between disks/post-coalescence mergers in the $\left[\sigma_{a}-v_{a}\right]$ plane changes from 0.135 to 0.096 .

The $\mathrm{W}-\left[\sigma_{a}-v_{a}\right]$ plane is less sensitive to resolution effects after redshifting our sample at $z=3$ (see Fig. 15). This is because the larger/outer regions are the ones less affected by resolution effects. As with this new criterion, the associated asymmetries weight more than those present at inner radii, the computed asymmetries (i.e., $v_{\text {asym }}, \sigma_{\text {asym }}$ ) are less affected by resolution. Therefore, the total kinematic asymmetry distinguishing the two classes changes only from 0.146 to 0.130 between $z=0$ and $z=3$.

Summarizing, resolution effects tend to smooth kinematic deviations making objects appear more disky than they actually are. These effects are more significant when analyzing the kinematic asymmetries in the (unweighted) $\left[\sigma_{a}-v_{a}\right]$ plane than in 
the $\mathrm{W}-\left[\sigma_{a}-v_{a}\right]$ one. In particular, when comparing our local seeing limited observations with simulated data at $z=3$ (and $0.1^{\prime \prime} /$ spaxel), the total kinematic asymmetry border value is reduced by a $30 \%$ from $z=0$ to $z=3$ in the $\left[\sigma_{a}-v_{a}\right]$ plane, while it is only shifted by $11 \%$ in the $\mathrm{W}-\left[\sigma_{a}-v_{a}\right]$ plane.

Comparing our results with those obtained in S08 when considering local spirals and toy-disk models as observed at high redshift, we note that the low value of our $K_{\text {tot }}(\sim 0.1)$ classifies those galaxies as mergers and, on the other hand, the frontier defined in S08 classifies our post-coalescence mergers and IRAS F12112+0305 as disks. This illustrates that the definition of a merger is a crucial point when defining the asymmetry frontier. A lower value of the fraction merger/disk (i.e., a higher value of the $K_{\text {tot }}$ parameter) can be derived when only pre-coalescence ongoing mergers are considered as "true" mergers. The weighted criterion proposed here should be applied to larger and more diverse samples in order to understand the uncertainties associated with this type of classifications.

\section{Conclusions}

We have presented our results for the spatially resolved kinematics of four local $(z \sim 0.016)$ luminous infrared galaxies (i.e., LIRGs) observed with the VLT/VIMOS IFU as part of a larger project to characterize the properties of (U)LIRGs based on optical and infrared IFS data. The four galaxies are at similar distances ( $\sim 70 \mathrm{Mpc})$ and, based on their morphologies, two of them have been classified as isolated disks and the other two as post-coalescence merger objects (see Paper III). We have analyzed the velocity field and velocity dispersion maps to study in detail their kinematics. The kinemetry method (developed by Krajnović and coworkers) is used to characterize the kinematic asymmetries of these galaxies and several criteria are discussed to distinguish their status. We draw the following conclusions from this study:

- The general kinematic properties of the four LIRGs are consistent with their morphological classification: isolated disks are found to have quite regular velocity fields and centrally peaked velocity dispersion maps consistent with a single rotating disk interpretation, while the remaining two galaxies have slightly different properties. In particular, we found that post-coalescence mergers have more irregular velocity fields and velocity dispersion maps that have off-nuclear velocity dispersion peaks (up to $220 \mathrm{~km} \mathrm{~s}^{-1}$ at $2.4 \mathrm{kpc}$ from the $\mathrm{H}_{\alpha}$ peak for IRAS F04315-0840) or nuclear asymmetric structures.

- We have found double-peaked emission-line profiles in the inner regions of the four galaxies. The secondary broad components (i.e., $\sigma \sim 70-450 \mathrm{~km} \mathrm{~s}^{-1}$ ) are in all cases blueshifted $\left(\Delta v \sim 50-150 \mathrm{~km} \mathrm{~s}^{-1}\right)$ and, taking into account the large velocities involved, they can be well explained by the presence of an outflow in a dusty environment. The pattern of the 2D kinematic maps of the secondary broad component for the two post-coalescence mergers (with kinematic axes perpendicular to those of the main component) further supports this interpretation. In the particular case of IRAS F04315-0840, the broad component is found over a quite extended area $(\sim 2.4 \mathrm{kpc} \times 2.7 \mathrm{kpc})$.

- Our measured values of the $v_{\mathrm{c}} / \sigma_{\mathrm{c}}$ parameter classify our sources as rotation dominated. Similar results are obtained when using the quantity $v_{\text {shear }} / \Sigma$. This shows that our postcoalescence mergers have a large rotation component and that the sole use of these parameters does not allow us to discriminate their kinematic differences from those of disks.

- When the full 2D kinematics information provided by the spatially resolved velocity field and velocity dispersion maps is considered, the kinematic asymmetries are well characterized with kinemetry, making the morphological and kinematic classification consistent for the four objects. Disks have lower kinematic asymmetries than those derived for post-coalescence mergers.

- We have explored a new criterion to characterize the kinematic asymmetries using kinemetry. In particular, we introduce a new weighting method that gives weight to the kinematics of the outer regions when computing the total asymmetries $v_{\text {asym }}$ and $\sigma_{\text {asym }}$. This step is important because post-coalescence mergers have relatively small kinematic asymmetries in the inner parts as a consequence of the rapid relaxation into a rotating disk, with the outer parts being still out of equilibrium (i.e., having larger asymmetries). The "frontier" between disks and post-coalescence systems only changes by $11 \%$ when considering the local and high$z$ cases suggests that this new criterion dependents less on angular resolution effects. Thus, the $\mathrm{W}-\left[\sigma_{a}-v_{a}\right]$ plane differentiates in a more robust way disks from post-coalescence mergers.

- Classifying disk/merger systems using kinemetry is difficult, and clearly depends on the definition of a merger. The "asymmetry frontier" strongly depends on the "type" of mergers considered: if only pre-coalescence ongoing mergers are considered as "true" mergers, a lower value of the fraction of merger/disk systems can be derived. The weighted criterion proposed here helps us to characterize in a more robust way post-coalescence asymmetries.

- If we had used previously defined criteria to classify disks and mergers, our two post-coalescence systems would have been classified as disks. This suggests that the number of mergers to disk systems at high- $z$ may have been underestimated. Larger and more diverse samples are required to confirm this conclusion.

Acknowledgements. We acknowledge the anonymous referee for useful comments and suggestions, that helped us to improve the quality of the paper. We also would like to thank Davor Krajnović for his help and valuable comments on his Kinemetry software. This work was funded in part by the Marie Curie Initial Training Network ELIXIR of the European Commission under contract PITN-GA-2008-214227. This work has been supported by the Spanish Ministry of Science and Innovation (MICINN) under grant ESP2007-65475-C02-01. Based on observations carried out at the European Southern observatory, Paranal (Chile), Programs 076.B-0479(A), 078.B-0072(A) and 081.B-0108(A). This research made use of the NASA/IPAC Extragalactic Database (NED), which is operated by the Jet Propulsion Laboratory, California Institute of Technology, under contract with the National Aeronautic and Space Administration.

\section{Appendix A: Other kinemetry-based criteria for detecting asymmetries}

We describe other criteria that could be useful in distinguishing different systems. Other authors have indeed tried to define criteria able to kinematically classify galaxies at intermediate redshift (i.e., $z \sim 0.6$ ): for example, Flores et al. (2006) developed a simple kinematic classification scheme for distant galaxies based on their 3D kinematics and their morphology in ACS F775W images. They modeled the velocity field of a rotating disk that matches the observed velocity gradient to obtain the expected $\sigma$-map corresponding to the observed velocity field and then compare the observed and model $\sigma$-maps, estimating whether the observed kinematics are consistent with a rotating disk (i.e., 

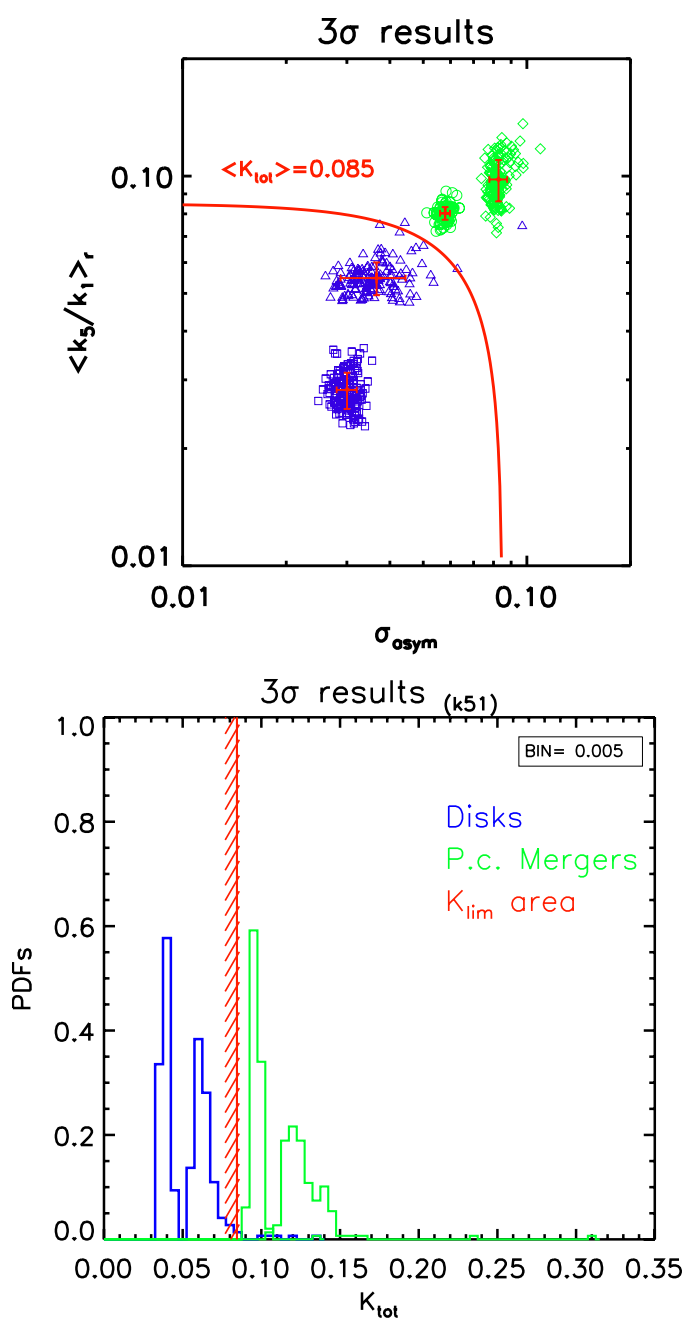

Fig. A.1. Top: asymmetry measures of the $\left\langle k_{5} / k_{1}\right\rangle$ term and velocity dispersion $\sigma_{\text {asym }}^{(w)}$ as derived from the MC realizations for the four objects. For each source, $150 \mathrm{MC}$ simulations are run but the $\pm 3 \sigma$ results are shown. The solid red line indicates the empirical division between disks and mergers at $\left\langle K_{\lim }\right\rangle=0.085$. Bottom: the probability distribution function (PDFs) derived from the respective MC realizations. The empirical delineation clearly separate the two classes defining a small range of possible values (i.e., $K_{\text {lim }}$ area). Small deviations $\left\langle k_{5} / k_{1}\right\rangle$ are found for disks, while higher values are found for mergers, which is consistent with our expectation.

a rotating disk should show a well-defined peak in the center of the $\sigma$ map). The spatial separation ( $\Delta r$, in pixels) between the peaks in the two $\sigma$-maps and the relative difference $(\epsilon)$ between the amplitudes of the modeled and observed $\sigma$ peaks are considered to classify the objects. They found that rotating disks have locations near $\Delta r \sim \epsilon \sim 0$, while galaxies with anomalous velocity fields are clearly offset. The same criterion was applied in Yang et al. (2008).

Here we propose some additional criteria, based again on the kinemetry method:

1. We consider the normalized higher-order deviations $\left\langle k_{5} / k_{1}\right\rangle_{v}$ of the velocity field with respect to those of the velocity dispersion map $\sigma_{\text {asym }}$, since the $k_{5}$ term indicates complex structure in the velocity map, as noted in Krajnović et al. (2006). Small $k_{5} / k_{1}$ values should be found in disk-like structures, while large $k_{5} / k_{1}$ are expected for mergers, where more complex structures are assumed to exists. This is confirmed by our results: class 0 objects have $\left\langle k_{5} / k_{1}\right\rangle$ close to zero, the

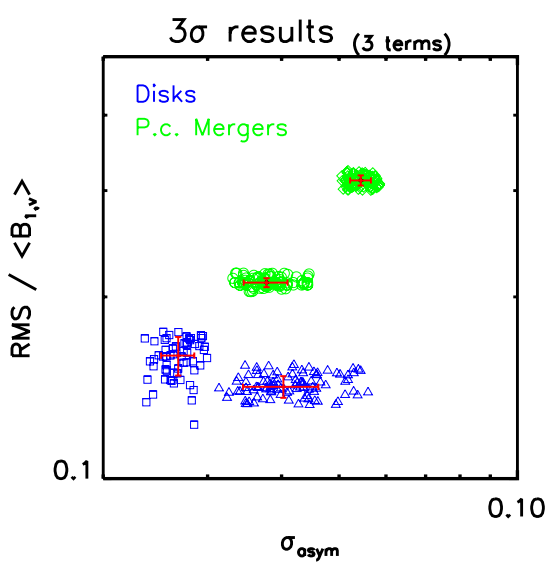

Fig. A.2. Subtracting the circular motion (i.e., $B_{1}$ coefficient) from the data, we end up with the velocity residual maps, characterized by mean residuals close to zero and an rms smaller than $20 \mathrm{~km} \mathrm{~s}^{-1}$ for the whole sample. If we assume that the four objects have almost the same rms, this plot clearly implies higher circular velocities for the class 0 galaxies, while we note that a lower $\mathrm{B}_{1}$ term is derived for the class 2 ones. Their rotation is clearly shown in Figs. 5-8.

amplitude of $k_{1}$ is substantial, and the position angle $\Gamma$ and flattening $q$ remain quite constant such that they can be classified as disk-like objects. On the other hand, class 2 objects show a rise in the $\left\langle k_{5} / k_{1}\right\rangle$ term of up to 0.6 at outer radii, where peculiar structures can be identified. In Fig. A.1, we present the results obtained from our MC simulations (150 for each object). This criterion allows us to distinguish the class $0 / 2$ in a similar way as in the $\left[\sigma_{a}-v_{a}\right]$ plane, but somewhat worst than in $\mathrm{W}-\left[\sigma_{a}-v_{a}\right]$ plane.

2. In some cases, the data quality does not allow us to apply sophisticated criteria to search for asymmetries. In these cases, a simple criterion may be the only possible option. Therefore, we have studied the potential of the simple$\left[\sigma_{a}-v_{a}\right]$ plane (hereafter, $S-\left[\sigma_{a}-v_{a}\right]$ ), where only the firstorder correction in the harmonic expansion is considered and asymmetries are defined as the ratio of the root mean square (rms) of the residual map to the mean values of the $B_{1, v}$ term over all the radii. The next formula describes the two asymmetries

$v_{\text {asym }}=\frac{\mathrm{rms}}{\left\langle B_{1, v}\right\rangle_{r}} \quad \sigma_{\text {asym }}=\left\langle\frac{k_{1, \sigma}}{B_{1, v}}\right\rangle_{r}$

where the rms gives an estimate of the deviations of the system from the ideal case of simple rotation in the system ${ }^{7}$. In Fig. A.2, we present the results in the $S-\left[\sigma_{a}-v_{a}\right]$ plane. Comparing this plot with that shown in Fig. 9, we note that the rms $/\left\langle B_{1}\right\rangle$ term tends to be higher than the $v_{\text {asym }}$ values, while the $\sigma_{\text {asym }}^{3 \text { terms }}$ values are smaller than the $\sigma_{\text {asym }}^{10 \text { terms }}$ range. The $\sigma_{\text {asym }}^{3 \text { terms }}$ does not seem to clearly characterize the kinematic asymmetries of these systems (i.e., in the case of IRAS F04315-0840, which has a quite distorted morphology). On the other hand, the rms $/\left\langle B_{1}\right\rangle$ term by itself can quite clearly distinguish the two classes, where a border dividing them can be estimated to have a value of $\sim 0.2$.

\footnotetext{
7 Residuals are derived from the measured velocities and the $\mathrm{B}_{1}$ coefficient.
} 
E. Bellocchi et al.: Kinematic asymmetries of disks and post-coalescence mergers

\section{References}

Arribas, S., Bushouse, H., Lucas, R. A., Colina, L., \& Borne, K. D. 2004, AJ, 127,2522

Arribas, S., Colina, L., Monreal-Ibero, A., et al. 2008, A\&A, 479, 687

Basu-Zych, A. R., Gonçalves, T. S., Overzier, R., et al. 2009, ApJ, 699, L118

Blain, A. W., Smail, I., Ivison, R. J., Kneib, J.-P., \& Frayer, D. T. 2002,

Phys. Rep., 369, 111

Bournaud, F., Chapon, D., Teyssier, R., et al. 2011, ApJ, 730, 4

Colina, L., Arribas, S., \& Monreal-Ibero, A. 2005, ApJ, 621, 725

Combes, F., Rampazzo, R., Bonfanti, P. P., Prugniel, P., \& Sulentic, J. W. 1995, A\&A, 297, 37

Corbett, E. A., Kewley, L., Appleton, P. N., et al. 2003, ApJ, 583, 670

Dekel, A., Sari, R., \& Ceverino, D. 2009, ApJ, 703, 785

Dib, S., Bell, E., \& Burkert, A. 2006, ApJ, 638, 797

Epinat, B. 2011, in Tracing the Ancestry of Galaxies (on the land of our ancestors), Proceedings of the International Astronomical Union, IAU Symp., 277, 128

Epinat, B., Amram, P., Balkowski, C., \& Marcelin, M. 2010, MNRAS, 401, 2113

Flores, H., Hammer, F., Puech, M., Amram, P., \& Balkowski, C. 2006, A\&A, 455,107

Förster Schreiber, N. M., Genzel, R., Lehnert, M. D., et al. 2006, ApJ, 645, 1062

Förster Schreiber, N. M., Genzel, R., Bouché, N., et al. 2009, ApJ, 706, 1364

García-Marín, M., Colina, L., Arribas, S., \& Monreal-Ibero, A. 2009, A\&A, 505, 1319

Genzel, R., Tacconi, L. J., Rigopoulou, D., Lutz, D., \& Tecza, M. 2001, ApJ, 563, 527

Genzel, R., Tacconi, L. J., Eisenhauer, F., et al. 2006, Nature, 442, 786

Genzel, R., Burkert, A., Bouché, N., et al. 2008, ApJ, 687, 59

Gonçalves, T. S., Basu-Zych, A., Overzier, R., et al. 2010, ApJ, 724, 1373

Hammer, F., Flores, H., Elbaz, D., et al. 2005, A\&A, 430, 115

Hinz, J. L., \& Rieke, G. H. 2006, ApJ, 646, 872

Jesseit, R., Naab, T., Peletier, R. F., \& Burkert, A. 2007, MNRAS, 376, 997
Krajnović, D., Cappellari, M., de Zeeuw, P. T., \& Copin, Y. 2006, MNRAS, 366, 787

Kronberger, T., Kapferer, W., Schindler, S., \& Ziegler, B. L. 2007, A\&A, 473, 761

Law, D. R., Steidel, C. C., Erb, D. K., et al. 2009, ApJ, 697, 2057

Le Fèvre, O., Saisse, M., Mancini, D., et al. 2003, in SPIE Conf. 4841, ed. M. Iye, \& A. F. M. Moorwood, 1670

Le Floc'h, E., Papovich, C., Dole, H., et al. 2005, ApJ, 632, 169

Lemoine-Busserolle, M., \& Lamareille, F. 2010, MNRAS, 402, 2291

Lemoine-Busserolle, M., Bunker, A., Lamareille, F., \& Kissler-Patig, M. 2010, MNRAS, 401, 1657

Maíz-Apellániz, J. 2004, PASP, 116, 859

Monreal-Ibero, A., Arribas, S., Colina, L., et al. 2010, A\&A, 517, A28

Pérez-González, P. G., Rieke, G. H., Egami, E., et al. 2005, ApJ, 630, 82

Pérez-González, P. G., Rieke, G. H., Villar, V., et al. 2008, ApJ, 675, 234

Puech, M. 2010, MNRAS, 406, 535

Rodríguez Zaurín, J., Tadhunter, C. N., \& González Delgado, R. M. 2010, MNRAS, 403, 1317

Rodríguez-Zaurín, J., Arribas, S., Monreal-Ibero, A., et al. 2011, A\&A, 527, A60

Rujopakarn, W., Rieke, G. H., Eisenstein, D. J., \& Juneau, S. 2011, ApJ, 726, 93

Sanders, D. B., \& Mirabel, I. F. 1996, ARA\&A, 34, 749

Sanders, D. B., Mazzarella, J. M., Kim, D.-C., Surace, J. A., \& Soifer, B. T. 2003, AJ, 126, 1607

Shapiro, K. L., Genzel, R., Förster Schreiber, N. M., et al. 2008, ApJ, 682, 231

Spitzer, L. 1987, Dynamical evolution of globular clusters (Princeton University Press)

Tacconi, L. J., Genzel, R., Lutz, D., et al. 2002, ApJ, 580, 73

Tacconi, L. J., Neri, R., Chapman, S. C., et al. 2006, ApJ, 640, 228

Tacconi, L. J., Genzel, R., Smail, I., et al. 2008, ApJ, 680, 246

Väisänen, P., Mattila, S., Kniazev, A., et al. 2008, MNRAS, 384, 886

Veilleux, S., Kim, D.-C., Sanders, D. B., Mazzarella, J. M., \& Soifer, B. T. 1995, ApJS, 98, 171

Veilleux, S., Kim, D.-C., \& Sanders, D. B. 2002, ApJS, 143, 315

Yang, Y., Flores, H., Hammer, F., et al. 2008, A\&A, 477, 789 